\title{
INVESTIGATION OF THE NEUTRALINO PAIR PRODUCTION AT LHC
}

\author{
A. I. Ahmadov $1,2 * *$ I. Boztosun ${ }^{1}$, R. Kh. Muradov ${ }^{2}$, \\ A. Soylu ${ }^{1}$, and E. A. Dadashov ${ }^{2}$ \\ ${ }^{1}$ Department of Physics, Faculty of Arts and Sciences, \\ Erciyes University, Kayseri, Turkey \\ ${ }^{2}$ Baku State University, Z. Khalilov st. 23, AZ-1148, Baku, Azerbaijan
}

(Dated: May 28, 2018)

\begin{abstract}
In this article, we investigate the Drell-Yan process of the light neutralino pair $\widetilde{\chi}_{i}^{0} \widetilde{\chi}_{j}^{0}(i, j=1,2)$ productions at proton-proton collisions and we present the general formulae for the differential cross sections. We conduct an extensive examination of the dependence of the total cross section of the subprocesses $q \bar{q} \rightarrow \widetilde{\chi}_{i}^{0} \widetilde{\chi}_{j}^{0}$ on the beam energy, on the mass of the squarks and also on the $M_{2}$ gaugino for the three extremely different scenarios. For all three cases, the outcomes are as follows: The dependence of the total cross section of the subprocesses $q \bar{q} \rightarrow \widetilde{\chi}_{i}^{0} \widetilde{\chi}_{j}^{0}$ on the beam energy is dominated by one of the subprocesses, $q \bar{q} \rightarrow \widetilde{\chi}_{1}^{0} \widetilde{\chi}_{2}^{0}$. On the other hand, the dependence of the total cross section of the subprocesses $q \bar{q} \rightarrow \widetilde{\chi}_{i}^{0} \widetilde{\chi}_{j}^{0}$ on the mass of the squarks is dominated by one of the subprocesses, $q \bar{q} \rightarrow \widetilde{\chi}_{1}^{0} \widetilde{\chi}_{1}^{0}$. We derive there from that our findings may lead to new insights relating to experimental investigations and these dependencies may be used as bases of an experimental research for the neutralino pair at LHC.
\end{abstract}

PACS numbers: 11.30.Pb, 12.15.-y, 12.60.Jv, 14.80.Ly,

Keywords: chargino sector, neutralino production, cross section

*Electronic address: E-mail:ahmadovazar@yahoo.com 


\section{INTRODUCTION}

The Standard Model (SM) is a successful theory of strong and electroweak interactions up to the energies accessible at present [1]. The hierarchy problem suggests that, in principle, SM is one of the fundamental effective theories of the low energy region. Supersymmetry (SUSY) is presently the most popular attempt to solve the hierarchy problem of SM, where the cancelletion of quadratic divergences is guaranteed and hence any mass scale is stable under radiative corrections. The most favorable candidate for a realistic extension of SM is the minimal supersymmetric standard model (MSSM). In MSSM, a discrete symmetry called R-parity $[2,3]$ is kept in order to assure baryon and lepton number conservations since the gauge-coupling unification supports conservation of R-parity. The minimal supersymmetric standard model (MSSM) [4] predicts that there exists an absolutely stable LSP. Most often the LSP in the MSSM theory is the lightest Majorana fermionic neutralino $\widetilde{\chi}_{1}^{0}$. Therefore the production of the lightest neutralino $\widetilde{\chi}_{1}^{0}$ and the second lightest neutralino $\widetilde{\chi}_{2}^{0}$ may be studied at present and future experiments and the detailed study of the neutralino sector will help us to determine which kind of the supersymmetric models really exists in nature. They are determined by diagonalizing the corresponding mass matrix. In MSSM, the mass matrix depends on four unknown parameters, namely $\mu, M_{2}, M_{1}$, and $\tan \beta=v_{2} / v_{1}$, which is the ratio of the vacuum expectation values of the two Higgs fields. $\mu$ is the supersymmetric Higgs-boson mass parameter and $M_{2}$ and $M_{1}$ are the gaugino mass parameters associated with the $S U(2)$ and $U(1)$ subgroups, respectively. The direct search of supersymmetric particles 
in experimental research is one of the promising tasks for present and future colliders. The multi-TeV Large Hadron Collider (LHC) at CERN and the possible future Next Linear Collider (NLC) are elaborately designed in order to study the symmetry-breaking mechanism and the new physics beyond SM. If the supersymmetry really exists at TeV scale, SUSY particles should be discovered and it will be possible to make accurate measurements to determine their masses and other parameters of the Lagrangian at LHC, and then we will have a better understanding of the supersymmetry model. We know that there are several mechanisms inducing the production of a neutralino/chargino pair at hadron colliders. One is through the quark-antiquark annihilation, called the Drell-Yan process, and another is via gluon-gluon fusion. Although the antiquark luminosity in the distribution function of the proton is much lower than gluon, the cross sections of the neutralino pair productions via the Drell-Yan mechanism are competitive with those from the gluon-gluon fusion, since the former mechanism of the neutralino pair productions is accessible at the tree level. These facts make the production rates in the Drell-Yan process competitive with or even larger than those in gluon-gluon fusions. But, the reactions $q \bar{q} \rightarrow \widetilde{\chi}_{i}^{0} \widetilde{\chi}_{j}^{0}$ are only subprocesses of the parent $p p$ hadron collider. In work [5], the authors have considered the production of neutralino pair at a high energy hadron collider, putting a special emphasis on the case where one of them is the lightest neutralino $\widetilde{\chi}_{1}^{0}$, possibly constituting the main Dark Matter component. Neutralino pair production in proton-proton collisions have been studied in [6] as well, but, our results disagree with those conducted in Ref. [6]. In our calculations, we have used the anticommuting nature of the Fermionic fields in amplitude 
and cross section of processes, which do not agree with the fermionic symmetry property assumed in Ref. [6]. Therefore, this approach is significant for the theoretical and experimental studies relating to the neutralino pair productions through the proton-proton collisions at LHC. These results imply an interesting complementarity between the future LHC measurements and the related $\gamma \gamma \rightarrow \widetilde{\chi}_{i}^{0} \widetilde{\chi}_{j}^{0}$ measurements at a future Linear Collider. Within this context, this paper is organized as follows: in section III, we present some formulae for the neutralino/chargino sector. In section III, we provide the formulae for the amplitudes and the differential cross sections of subprocesses $q \bar{q} \rightarrow \widetilde{\chi}_{i}^{0} \widetilde{\chi}_{j}^{0}$ and in section $\llbracket$, we present the numerical results for the cross-section and discuss the dependence cross-section on the SUSY model parameters. We state our conclusions in section $\mathrm{V}$,

\section{MSSM PARAMETERS IN NEUTRALINO/CHARGINO SECTOR}

In the minimal supersymmetric extension of the Standard Model (MSSM), the physical neutralino mass eigenstates $\chi_{1}^{0}(\mathrm{i}=1,2,3,4)$ are the combinations

of the neutral gauginos ( $\widetilde{B}$ and $\widetilde{W}^{3}$ ) and the neutral higgsinos $\left(\widetilde{H}_{1}^{0}, \widetilde{H}_{2}^{0}\right)$. In the two-component fermion fields $\psi_{j}^{0}=\left(-i \lambda^{1},-i \lambda^{3}, \psi_{H_{1}^{0}}, \psi_{H_{2}^{0}}\right)[4,7]$, where $\lambda^{1}$ is the bino and $\lambda^{3}$ is the neutral wino, the neutralino mass term in the Lagrangian is given by

$$
L=-\frac{1}{2}\left(\psi^{0}\right)^{T} M \psi^{0}+\text { h.c. }
$$


The neutralino mass matrix $[4,7]$ in the $\left(\widetilde{B}, \widetilde{W}, \widetilde{H}_{1}^{0}, \widetilde{H}_{2}^{0}\right)$ basis,

$$
M=\left(\begin{array}{cccc}
M_{1} & 0 & -m_{Z} c_{\beta} s_{W} & m_{Z} s_{\beta} s_{W} \\
0 & M_{2} & m_{Z} c_{\beta} c_{W} & -m_{Z} s_{\beta} c_{W} \\
-m_{Z} c_{\beta} s_{W} & m_{Z} c_{\beta} c_{W} & 0 & -\mu \\
m_{Z} s_{\beta} s_{W} & -m_{Z} s_{\beta} c_{W} & -\mu & 0
\end{array}\right)
$$

is built up by the fundamental supersymmetry parameters: the $U(1)$ and $S U$ (2) gaugino masses $M_{1}$ and $M_{2}$, the higgsino mass parameter $\mu$, and the ratio $\tan \beta=v_{2} / v_{1}$ of the vacuum expectation values of the two neutral Higgs fields, which break the electroweak symmetry. Here, $s_{\beta}=\sin \beta, c_{\beta}=$ $\cos \beta$ and $s_{W}, c_{W}$ are the sine and cosine of the electroweak mixing angle $\theta_{W}$. In CP-noninvariant theories, the mass parameters are complex. By the reparametrization of the fields, $M_{2}$ can be taken as real and positive without loss of generality so that the two remaining nontrivial phases, which are reparametrization-invariant, may be attributed to $M_{1}$ and $\mu$ :

$$
M_{1}=\left|M_{1}\right| e^{i \phi_{1}} \quad \text { and } \quad \mu=|\mu| e^{i \phi_{\mu}}\left(0 \leq \phi_{1}, \phi_{\mu}<2 \pi\right)
$$

The experimental analysis of neutralino properties in production and decay mechanisms will unravel the basic structure of the underlying supersymmetries theory. The charginos $\tilde{\chi}_{j}^{+}(j=1,2)$ mass matrix in the current eigenstate basis have the form [7]

$$
M_{c}=\left(\begin{array}{cc}
M_{2} & \sqrt{2} m_{W} c_{\beta} \\
\sqrt{2} m_{W} s_{\beta} & |\mu| e^{i \phi_{\mu}}
\end{array}\right)
$$


is diagonalized by two different unitary matrices $U_{R} M_{c} U_{L}^{+}=\operatorname{diag}\left\{m_{1}^{ \pm}, m_{2}^{ \pm}\right\}$, parametrized in general by two rotation angles and four phases:

$$
U_{L}=\left(\begin{array}{cc}
c_{L} & s_{L}^{\star} \\
-s_{L} & c_{L}
\end{array}\right)
$$

and

$$
U_{R}=\operatorname{diag}\left\{e^{i \gamma_{1}}, e^{i \gamma_{2}}\right\} \cdot\left(\begin{array}{cc}
c_{R} & s_{R}^{\star} \\
-s_{R} & c_{R}
\end{array}\right)
$$

where $c_{L, R}=\cos \phi_{L, R} \quad$ and $s_{L, R}=\sin \phi_{L, R} e^{i \delta_{L, R}}$. In the limit of $M_{2}^{2},|\mu|^{2} \gg$ $m_{Z}^{2}$ and $\left|M_{2} \pm\right| \mu||^{2} \gg m_{Z}^{2}$, the following expressions

$$
\begin{gathered}
m_{1}^{ \pm}=M_{2}+X_{2}\left[M_{2}+|\mu| s_{2 \beta} \cos \phi_{\mu}\right], \\
m_{2}^{ \pm}=|\mu| X_{2}\left[|\mu|+M_{2} s_{2 \beta} \cos \phi_{\mu}\right],
\end{gathered}
$$

are found for the chargino masses and

$$
\begin{gathered}
s_{L}=\frac{\sqrt{2} m_{W}}{M_{2}^{2}-|\mu|^{2}}\left(M c_{\beta}+\mu^{\star} s_{\beta}\right) \quad \gamma_{1}=+X_{2} \frac{|\mu|}{M_{2}} s_{2 \beta} \sin \phi_{\mu} \\
s_{R}=\frac{\sqrt{2} m_{W}}{M_{2}^{2}-|\mu|^{2}}\left(\mu c_{\beta}+M_{2}^{\star} s_{\beta}\right) \quad \gamma_{2}=-X_{2} \frac{M_{2}}{|\mu|} s_{2 \beta} \sin \phi_{\mu} \\
X_{2}=\frac{m_{Z}^{2} c_{W}^{2}}{\left|M_{2}\right|^{2}-|\mu|^{2}}
\end{gathered}
$$

for the mixing angles and phases. In the present work, we have investigated the Drell-Yan process of the light neutralino pair $\widetilde{\chi}_{i}^{0} \widetilde{\chi}_{j}^{0}(i, j=1,2)$ productions at hadron colliders. Since the neutralino mass matrix $M$ is symmetric, one unitary matrix $N$ is sufficient to rotate the gauge eigenstate basis $\left(\widetilde{B}^{0}, \widetilde{W}^{3}, \widetilde{H}_{1}^{0}, \widetilde{H}_{2}^{0}\right)$ to the mass eigenstate basis of the Majorana fields $\widetilde{\chi}_{i}^{0}$.

$$
M_{D}=N^{T} M N=\sum_{j=1}^{4} m_{\widetilde{\chi}_{j}^{0}} E_{j},
$$


where $N$ is a unitary matrix. To determine $N$, it is easiest to square eq.(2.1) obtaining

$$
M_{D}^{2}=N^{-1} M^{+} M N=\sum_{j=1}^{4} m_{\tilde{\chi}_{j}^{0}}^{2} E_{j},
$$

where $\left(E_{j}\right)_{4 x 4}$ are the basic matrices defined by $\left(E_{j}\right)_{i k}=\delta_{j i} \delta_{j k}$ and $\widetilde{\chi}_{j}^{0}$ stand for the four component Majorana neutralinos:

$$
\widetilde{\chi}_{j}^{0}=\left(\begin{array}{c}
\widetilde{\chi}_{j}^{0} \\
\ldots \\
\widetilde{\chi}_{j}^{0}
\end{array}\right), j=1, \ldots, 4
$$

Here, we suppose that the real eigenvalues of $M_{D}$ are ordered in the following way

$$
m_{\widetilde{\chi}_{1}^{0}} \leq m_{\widetilde{\chi}_{2}^{0}} \leq m_{\widetilde{\chi}_{3}^{0}} \leq m_{\widetilde{\chi}_{4}^{0}}
$$

The mass eigenvalues $m_{\tilde{\chi}_{j}^{0}}(j=1,2,3,4)$ in $M_{D}$ can be chosen as positive by a suitable definition of the unitary matrix $N$. In this work, we consider the higgsino/gaugino sector with the following assumptions: First, for simplification, $C P$-conservation is hold, namely $\phi_{\mu}=\phi_{1}=0$. The physical signs among $M_{1}, M_{2}$ and $\mu$ are relative, which can be absorbed into phases $\phi_{\mu}$ and $\phi_{1}$ by redefinition of fields. Thus, $M_{1}, M_{2}$ and $\mu$ are chosen to be real and positive, i.e., $M_{1}, M_{2}, \mu>0$. With the above assumptions, there are several scenario for the choice of the SUSY parameters for the investigation of the neutralino pair production in hadron collider. One can employ the scenario of taking $M_{1}, M_{2}, \mu$, and $\tan \beta$ as input parameters, and then get all the physical chargino and neutralino masses and the matrix elements of $U_{R}, U_{L}^{+}$and $N$ as outputs. Also, there are other alternative scenarios, such 
as the CP conserving mSUGRA scenario with five input parameters, namely $m_{1 / 2}, m_{0}, A_{0}, \mu$ and $\tan \beta$, where $m_{1 / 2}, m_{0}$ and $A_{0}$ are the universal gaugino mass, scalar mass at GUT scale and the trilinear soft breaking parameter in the superpotential respectively. From these five parameters, all the masses and couplings of the model are determined by the evolution from the GUT scale down to the low electroweak scale [8]. Since SUSY parameters should be extracted from the physical quantities, one can also choose an alternative way to diagonalize the mass matrix $M$, by taking any two physical chargino masses together with $\tan \beta$ as inputs. There are several scenarios about the choice of two chargino masses and $\tan \beta$ [10]. Also there are two possible scenario about the choice of $\tan \beta$ : scenario with small $\tan \beta(\tan \beta \approx 1 \div 3)$ and scenario with large $\tan \beta(\tan \beta \approx 30 \div 70)[9]$. In this work, we take two chargino masses $m_{\tilde{\chi}_{1,2}^{+}}$and scenario with small tan $\beta$ as inputs. In this way, the two fundamental SUSY parameters, $M_{2}$ and $\mu$ can be figured out from the chargino masses by using the following formula: For given $\tan \beta$, the fundamental SUSY parameters $M_{2}$ and $\mu$ can be derived from these two chargino masses [11]. The sum and differences of the chargino masses lead to the following equations involving $M_{2}$ and $\mu$ :

$$
\begin{gathered}
M_{2}^{2}+|\mu|^{2}=m_{\widetilde{\chi}_{1}^{+}}+m_{\widetilde{\chi}_{2}^{+}}-2 m_{W}^{2}, \\
M_{2}^{2}|\mu|^{2}-2 m_{W}^{2} \sin 2 \beta \cos \phi_{\mu} M_{2}|\mu|+\left(m_{W}^{4} \sin ^{2} 2 \beta-m_{\widetilde{\chi}_{1}^{+}}^{2} m_{\widetilde{\chi}_{2}^{+}}^{2}\right)=0 .
\end{gathered}
$$


The solution of (2.5) is given as:

$$
M_{2}|\mu|=m_{W}^{2} \cos \phi_{\mu} \sin 2 \beta \pm \sqrt{m_{\widetilde{\chi}_{1}^{+}}^{2} m_{\widetilde{\chi}_{2}^{+}}^{2}-m_{W}^{4} \sin ^{2} 2 \beta \sin ^{2} \phi_{\mu}} .
$$

From (2.4) and (2.6), one obtains the following solutions for $M_{2}$ and $\mu$ :

$$
\begin{aligned}
& 2 M_{2}^{2}=\left(m_{\widetilde{\chi}_{1}^{+}}^{2}+m_{\widetilde{\chi}_{2}^{+}}^{2}-2 m_{W}^{2}\right) \mp\left(\sqrt{\left(m_{\tilde{\chi}_{1}^{+}}^{2}+m_{\widetilde{\chi}_{2}^{+}}^{2}-2 m_{W}^{2}\right)^{2}-\Delta_{ \pm}}\right), \\
& 2|\mu|^{2}=\left(m_{\tilde{\chi}_{1}^{+}}^{2}+m_{\tilde{\chi}_{2}^{+}}^{2}-2 m_{W}^{2}\right) \pm\left(\sqrt{\left(m_{\tilde{\chi}_{1}^{+}}^{2}+m_{\tilde{\chi}_{2}^{+}}^{2}-2 m_{W}^{2}\right)^{2}-\Delta_{ \pm}}\right),
\end{aligned}
$$

with

$$
\begin{gathered}
\Delta_{ \pm}=4\left[m_{\tilde{\chi}_{1}^{+}}^{2} m_{\widetilde{\chi}_{2}^{+}}^{2}+m_{W}^{4} \cos 2 \phi_{\mu} \sin ^{2} 2 \beta \pm 2 m_{W}^{2} \cos \phi_{\mu} \sin 2 \beta\right. \\
\left.\sqrt{m_{\widetilde{\chi}_{1}^{+}}^{2} m_{\tilde{\chi}_{2}^{+}}^{2}-m_{W}^{4} \sin ^{2} 2 \beta \sin ^{2} \phi_{\mu}}\right]
\end{gathered}
$$

where the upper signs correspond to $M_{2}<|\mu|$ regime, and the lower ones to $M_{2}>|\mu|$. Therefore, for given $\tan \beta, M_{2}$ and $\mu$ can be determined in terms of the masses of the charginos $m_{\widetilde{\chi}_{1}^{+}}$and $m_{\widetilde{\chi}_{2}^{+}}$by using (2.7), and (2.8) from which one gets four solutions corresponding to different physical scenarios. For $\mu<M_{2}$, the lightest chargino has a stronger higgsino-like component and therefore is referred to as higgsino-like. The solution $\mu>M_{2}$, corresponding to the gaugino-like situation, can be readily obtained by the substitutions: $M_{2} \rightarrow \mu$, and $\mu \rightarrow \operatorname{sign}(\mu) M_{2}$. In this paper, we assume the GUT relation $[11,12]$

$$
M_{1}=\frac{5}{3} M_{2} \tan ^{2} \Theta_{W}
$$


Thus, the neutralino masses can be determined by solving the characteristic equation associated to this system, that is

$$
X^{4}-a X^{3}+b X^{2}-c X+d=0,
$$

where

$$
\begin{gathered}
a=M_{1}^{2}+2 \mu^{2}+M_{2}^{2}+2 m_{Z}^{2} \\
b=\left(\mu^{2}+m_{Z}^{2}\right)^{2}+M_{2}^{2}\left(M_{1}^{2}+2 \mu^{2}+2 m_{Z}^{2} s_{W}^{2}\right)+2 M_{1}^{2}\left(\mu^{2}+m_{Z}^{2} c_{W}^{2}\right)-2 \mu m_{Z}^{2} c_{W}^{2} M_{2} \sin 2 \beta \\
\times \cos \phi_{\mu}-2 m_{Z}^{2} s_{W}^{2} M_{1} \sin 2 \beta \cos \left(\phi_{\mu}+\phi_{1}\right) \\
c=\mu^{4} M_{1}^{2}+\mu^{2} m_{Z}^{4} \sin ^{2} 2 \beta+M_{1}^{2} m_{Z}^{2} c_{W}^{2}\left(2 \mu^{2}+m_{Z}^{2} c_{W}^{2}\right)+ \\
M_{2}^{2}\left(m_{Z}^{4} s_{W}^{4}+2 \mu^{2}\left(m_{Z}^{2} s_{W}^{2}+M_{1}^{2}\right)+\mu^{4}\right)-2 \mu m_{Z}^{2} s_{W}^{2} M_{1}\left(\mu^{2}+M_{2}^{2}\right) \sin 2 \beta \cos \left(\phi_{\mu}+\phi_{1}\right)+ \\
2 m_{Z}^{2} c_{W}^{2} M_{2}\left[m_{Z}^{2} M_{1} s_{W}^{2} \cos \phi_{1}-\mu\left(\mu^{2}+M_{1}^{2}\right) \cos \phi_{\mu} \sin 2 \beta\right] \\
d=m_{Z}^{4} c_{W}^{4} \mu^{2} M_{1}^{2} \sin { }^{2} 2 \beta+2 m_{Z}^{2} \mu^{2} M_{1} M_{2} c_{W}^{2}\left(m_{Z}^{2} s_{W}^{2} \sin 2 \beta \cos \phi_{1}-\mu M_{1} \cos \phi_{\mu}\right)+ \\
\mu^{2} m_{Z}^{2} s_{W}^{2} M_{2}^{2} \sin 2 \beta\left(m_{Z}^{2} s_{W}^{2} \sin 2 \beta-2 \mu M_{1} \cos \left(\phi_{1}+\phi_{\mu}\right)\right)+\mu^{4} M_{1}^{2} M_{2}^{2} .
\end{gathered}
$$

Solving Eq.(2.10), we get the exact analytic formulae for the neutralino masses

$$
\begin{aligned}
& m_{\widetilde{\chi}_{1}^{0}}^{2}, m_{\widetilde{\chi}_{2}^{0}}^{2}=\frac{a}{4}-\frac{f}{2} \mp \frac{1}{2} \sqrt{r-w-\frac{p}{4 f}}, \\
& m_{\widetilde{\chi}_{3}^{0}}^{2}, m_{\widetilde{\chi}_{4}^{0}}^{2}=\frac{a}{4}+\frac{f}{2} \mp \frac{1}{2} \sqrt{r-w+\frac{p}{4 f}},
\end{aligned}
$$

where

$$
\begin{gathered}
f=\sqrt{\frac{r}{2}+w} \\
w=\frac{q}{\left(3 \cdot 2^{1 / 3}\right)}+\frac{\left(2^{1 / 3} \cdot h\right)}{3 \cdot q}, \\
q=\left(k+\sqrt{k^{2}-4 h^{3}}\right)^{1 / 3}
\end{gathered}
$$




$$
\begin{gathered}
k=2 b^{3}-9 a b c+27 c^{2}+27 a^{2} d-72 b d, \\
h=b^{2}-3 a c+12 d, \\
p=a^{3}-4 a b+8 c, \\
r=\frac{a^{2}}{2}-\frac{4 b}{3} .
\end{gathered}
$$

Starting from Eq.(2.2), we get

$$
\left(M^{+} M\right) N-N M_{D}^{2}=0 .
$$

A more explicit form of this matrix equation is

$$
\begin{aligned}
& \left(A_{11}-m_{\widetilde{\chi}_{j}^{0}}^{2}\right) N_{1 j}+A_{12} N_{2 j}+A_{13} N_{3 j}+A_{14} N_{4 j}=0, \\
& A_{21} N_{1 j}+\left(A_{22}-m_{\widetilde{\chi}_{j}^{0}}^{2}\right) N_{2 j}+A_{23} N_{3 j}+A_{24} N_{4 j}=0, \\
& A_{31} N_{1 j}+A_{32} N_{2 j}+\left(A_{33}-m_{\widetilde{\chi}_{j}^{0}}^{2}\right) N_{3 j}+A_{34} N_{4 j}=0, \\
& A_{41} N_{1 j}+A_{42} N_{2 j}+A_{43} N_{3 j}+\left(A_{44}-m_{\widetilde{\chi}_{j}^{0}}^{2}\right) N_{4 j}=0,
\end{aligned}
$$


$j=1, \ldots, 4$, where $A_{i j}=\sum_{k=1}^{4} M_{k i}^{\star} M_{k j}$ :

$$
\begin{aligned}
& A_{11}=M_{1}^{2}+m_{Z}^{2} s_{W}^{2}, \\
& A_{12}=A_{21}=-m_{Z}^{2} s_{W} c_{W}, \\
& A_{13}=A_{31}=-M_{1} m_{Z} c_{\beta} s_{W}-\mu m_{Z} s_{W} s_{\beta}, \\
& A_{14}=A_{41}=M_{1} m_{Z} s_{\beta} s_{W}+\mu m_{Z} c_{\beta} s_{W}, \\
& A_{22}=M_{2}^{2}+m_{Z}^{2} c_{W}^{2}, \\
& A_{23}=A_{32}=M_{2} m_{Z} c_{\beta} c_{W}+\mu m_{Z} s_{\beta} c_{W}, \\
& A_{33}=\mu^{2}+m_{Z}^{2} c_{\beta}^{2}, \\
& A_{24}=A_{42}=-M_{2} m_{Z} s_{\beta} c_{W}-\mu m_{Z} c_{\beta} c_{W}, \\
& A_{34}=A_{43}=-m_{Z}^{2} s_{\beta} c_{\beta}, \\
& A_{44}=m_{Z}^{2} s_{\beta}^{2}+\mu^{2} .
\end{aligned}
$$

The diagonalizing matrix $N$ can be obtained by computing the eigenvectors corresponding to the eigenvalues given in Eq.(2.11). Indeed, by inserting a generic eigenvalue $m_{\widetilde{\chi}_{j}^{0}}$, into Eq.(2.14) and dividing each one of these equations by $N_{1 j}$, where it is assumed that $N_{1 j} \neq 0$, we get

$$
\begin{aligned}
& A_{12} \frac{N_{2 j}}{N_{1 j}}+A_{13} \frac{N_{3 j}}{N_{1 j}}+A_{14} \frac{N_{4 j}}{N_{1 j}}-m_{\tilde{\chi}_{j}^{0}}^{2}=-A_{11}, \\
& \left(A_{22}-m_{\tilde{\chi}_{j}^{0}}^{2}\right) \frac{N_{2 j}}{N_{1 j}}+A_{23} \frac{N_{3 j}}{N_{1 j}}+A_{24} \frac{N_{4 j}}{N_{1 j}}=-A_{21}, \\
& A_{32} \frac{N_{2 j}}{N_{1 j}}+\left(A_{33}-m_{\tilde{\chi}_{j}^{0}}^{2}\right) \frac{N_{3 j}}{N_{1 j}}+A_{34} \frac{N_{4 j}}{N_{1 j}}=-A_{31}, \\
& A_{42} \frac{N_{2 j}}{N_{1 j}}+A_{43} \frac{N_{3 j}}{N_{1 j}}+\left(A_{44}-m_{\tilde{\chi}_{j}^{0}}^{2}\right) \frac{N_{4 j}}{N_{1 j}}=-A_{41},
\end{aligned}
$$

Solving this system of equations, and taking into account the relation

$$
\left|N_{1 j}\right|^{2}+\left|N_{2 j}\right|^{2}+\left|N_{3 j}\right|^{2}+\left|N_{4 j}\right|^{2}=1,
$$


it yields the $N_{i j}$ matrix's component

$$
N_{i j}=\frac{\Delta_{i j}}{\Delta_{1 j}} \cdot \frac{\left|\Delta_{1 j}\right|}{\sqrt{\left|\Delta_{1 j}\right|^{2}+\left|\Delta_{2 j}\right|^{2}+\left|\Delta_{3 j}\right|^{2}+\left|\Delta_{4 j}\right|^{2}}},
$$

when, $i=1, \ldots 4$. Here,

$$
\Delta_{1 j}=\left|\begin{array}{ccc}
A_{22}-m_{\widetilde{\chi}_{j}^{0}}^{2} & A_{23} & A_{24} \\
A_{32} & A_{33}-m_{\widetilde{\chi}_{j}^{0}}^{2} & A_{34} \\
A_{42} & A_{43} & A_{44}-m_{\widetilde{\chi}_{j}^{0}}^{2}
\end{array}\right|
$$

and $\Delta_{i j}, i=2,3,4$, is formed from $\Delta_{1 j}$ by substituting the (i-1)th column by

$$
\left(\begin{array}{c}
-A_{21} \\
-A_{31} \\
-A_{41}
\end{array}\right)
$$

\section{CALCULATION OF THE CROSS SECTION}

The Neutralino pair productions, which can be produced via the collisions of quark and antiquarks in protons, can be expressed as

$$
q\left(p_{1}\right) \bar{q}\left(p_{2}\right) \rightarrow \widetilde{\chi}_{i}^{0}\left(k_{1}\right) \widetilde{\chi}_{j}^{0}\left(k_{2}\right)
$$

where $p_{1}$ and $p_{2}$ represent the momenta of the incoming quark and antiquark, and $k_{1}$ and $k_{2}$ denote the momenta of the two final state neutralinos, respectively. The Mandelstam invariant variables for subprocess (3.1) are defined as

$$
\hat{s}=\left(p_{1}+p_{2}\right)^{2}, \quad \hat{t}=\left(p_{1}-k_{1}\right)^{2}, \quad \hat{u}=\left(p_{1}-k_{2}\right)^{2} .
$$

The Feynman diagrams of the subprocess are shown in Fig.1. The relevant couplings of the supersymmetric particles are deduced from the following 
interaction Lagrangians of the Supersymmetric Standard Model [13]:

$$
\begin{gathered}
L_{Z^{0} \widetilde{\chi}_{i}^{0} \widetilde{\chi}_{j}^{0}}=\frac{1}{2} \frac{g}{\cos \Theta_{\mathrm{W}}} Z_{\mu} \overline{\widetilde{\chi}}_{i}^{0} \gamma^{\mu}\left(O_{i j}^{\prime \prime} L_{q} P_{L}+O_{i j}^{\prime \prime} R_{q} P_{R}\right) \widetilde{\chi}_{j}^{0}, \\
L_{Z^{0} q \bar{q}}=\frac{g}{\cos \Theta_{\mathrm{W}}} \bar{q} \gamma^{\mu}\left(L_{q} P_{L}+R_{q} P_{R}\right) q Z_{\mu}, \\
L_{q \widetilde{q} \widetilde{\chi}^{0}}=-\sqrt{2} g \bar{q}\left[a_{i}^{L}\left(\widetilde{q}_{n}\right) P_{L}+a_{i}^{R}\left(\widetilde{q}_{n}\right) P_{R}\right] \widetilde{\chi}_{i}^{0} \widetilde{q}_{n} .
\end{gathered}
$$

In Eqs.(3.3-3.5) $\widetilde{\chi}_{i}^{0}$ and $q$ are four-component spinor fields and $\widetilde{q}$ is the field of the squark. Furthermore, $g=e / \sin \Theta_{W}(e>0)$ is the weak coupling constant, $\Theta_{W}$ the Weinberg angle, $P_{R, L}=\frac{1}{2}\left(1 \pm \gamma^{5}\right)$, while the coupling constant $O_{i j}^{\prime \prime}, L_{q}, R_{q}$ and $a_{i}^{R, L}\left(\widetilde{q}_{n}\right)$ are given by

$$
\begin{gathered}
O_{i j}^{\prime \prime L}=\frac{1}{2}\left(N_{i 3} N_{j 3}^{\star}-N_{i 4} N_{j 4}^{\star}\right) \cos 2 \beta-\frac{1}{2}\left(N_{i 3} N_{j 4}^{\star}+N_{i 4} N_{j 3}^{\star}\right) \sin 2 \beta, \\
O_{i j}^{\prime \prime R}=-O_{i j}^{\prime \prime L \star} \\
L_{q}=2 I_{q}^{3}\left(1-2 \sin ^{2} \Theta_{W}\left|Q_{q}\right|\right), \quad R_{q}=-2 \sin ^{2} \Theta_{W} Q_{q},
\end{gathered}
$$

with $I_{q}^{3}, Q_{q}$ being the isospin and charge of the various $q_{L}$-quarks, and

$$
\begin{gathered}
a_{i}^{L}\left(\widetilde{u}_{L}\right)=-\frac{e}{3 \sqrt{2} s_{W} c_{W}}\left(N_{1 i} s_{W}+3 N_{2 i} c_{W}\right), \\
a_{i}^{R}\left(\widetilde{u}_{R}\right)=\frac{2 \sqrt{2} e}{3 c_{W}} N_{1 i}^{\star}, \\
a_{i}^{L}\left(\widetilde{d}_{L}\right)=-\frac{e}{3 \sqrt{2} s_{W} c_{W}}\left(N_{1 i} s_{W}-3 N_{2 i} c_{W}\right), \\
a_{i}^{R}\left(\widetilde{d}_{R}\right)=-\frac{\sqrt{2} e}{3 c_{W}} N_{1 i}^{\star},
\end{gathered}
$$




$$
\begin{aligned}
& a_{i}^{L}\left(\widetilde{u}_{R}\right)=-\frac{e m_{u}}{\sqrt{2} m_{W} s_{W} s_{\beta}} N_{4 i}, \\
& a_{i}^{R}\left(\widetilde{u}_{R}\right)=-\frac{e m_{u}}{\sqrt{2} m_{W} s_{W} s_{\beta}} N_{4 i}^{\star}, \\
& a_{i}^{L}\left(\widetilde{d}_{R}\right)=-\frac{e m_{d}}{\sqrt{2} m_{W} s_{W} c_{\beta}} N_{3 i}, \\
& a_{i}^{R}\left(\widetilde{d}_{L}\right)=-\frac{e m_{d}}{\sqrt{2} m_{W} s_{W} c_{\beta}} N_{3 i}^{\star} .
\end{aligned}
$$

In (3.9), $(q=u, d)$ refer to the incoming up and down quark (antiquark) of any family, while $\left(\widetilde{q}_{n}=\widetilde{q}_{L}, \widetilde{q}_{R}\right)$ denote the corresponding squarks. We also note that the mixing matrices $\mathrm{N}$ in $(3.6,3.9)$, control the Bino, Wino, Higgsino components of the neutralino in the $Z \widetilde{\chi}_{i}^{0} \widetilde{\chi}_{j}^{0}$ and $q \widetilde{q} \widetilde{\chi}^{0}$ coupling. The corresponding Lorentz invariant matrix element for each of the diagrams can be written as

$$
T=T_{\hat{s}}+T_{\hat{t}}+T_{\hat{u}}
$$

where

$$
\begin{gathered}
T_{\hat{s}}=-\frac{e^{2}}{2 \sin ^{2} \Theta_{W} \cos ^{2} \Theta_{W}} D_{Z}(\hat{s}) \bar{u}_{i}\left(k_{1}\right) \gamma \mu\left[O_{Z}^{i j} P_{L}-O_{Z}^{i j \star} P_{R}\right] \vartheta_{j}\left(k_{2}\right) \times \\
\bar{v}\left(p_{2}\right) \gamma_{\mu}\left(g_{V_{q}}+g_{A_{q}} \gamma_{5}\right) u\left(p_{1}\right), \\
T_{\hat{t}}=\sum_{n} \frac{1}{\hat{t}-m_{\widetilde{q}_{n}}^{2}} \bar{u}_{i}\left(k_{1}\right)\left(a_{i}^{L}\left(\widetilde{q}_{n}\right) P_{L}+a_{i}^{R}\left(\widetilde{q}_{n}\right) P_{R}\right) u\left(p_{1}\right) \bar{v}\left(p_{2}\right) \times \\
T_{\hat{u}}=-\sum_{n} \frac{1}{\hat{u}-m_{\widetilde{q}_{n}}^{2}} \bar{u}_{j}\left(k_{2}\right)\left(a_{j}^{L \star}\left(\widetilde{q}_{n}\right) P_{R}+a_{j}^{R \star}\left(\widetilde{q}_{n}\right) P_{L}\right) u\left(p_{1}\right) \bar{v}\left(p_{2}\right) \times \\
\left(a_{i}^{L}\left(\widetilde{q}_{n}\right) P_{L}+a_{i}^{R}\left(\widetilde{q}_{n}\right) P_{R}\right) v_{i}\left(k_{1}\right),
\end{gathered}
$$

where the index $n$ refers to the summation over the exchanged $L$ - and $R$ squarks of the same flavor in the $t$-and $u$ - channel, and $(i, j)$ describe the final 
neutralinos. From the total (gauge-invariant) amplitude $T$, which is the sum of the partial amplitudes Eq.(3.11), we obtain the differential cross section as

$$
\frac{d \sigma}{d \Omega}=\frac{\lambda_{i j}}{384 \pi^{2} \hat{s}^{2}}\left(\frac{1}{2}\right)^{\delta_{i j}}\left(M_{\hat{s} \hat{s}}+M_{\hat{t} \hat{t}}+M_{\hat{u} \hat{u}}-2 M_{\hat{s} \hat{t}}+2 M_{\hat{s} \hat{u}}-2 M_{\hat{t} \hat{u}}\right),
$$

where

$$
\lambda_{i j}=\sqrt{\left(\hat{s}-m_{\widetilde{\chi}_{i}^{0}}^{2}-m_{\widetilde{\chi}_{j}^{0}}^{2}\right)^{2}-4 m_{\widetilde{\chi}_{i}^{0}}^{2} m_{\widetilde{\chi}_{j}^{0}}^{2}} / 2,
$$

and $\left(\frac{1}{2}\right)^{\delta_{i j}}$ is the final identical-particle factor. The squares of the matrix element have the form as

$$
\begin{aligned}
& M_{\hat{s} \hat{s}}=\frac{e^{4}}{4 \sin ^{4} \Theta_{W} \cos ^{4} \Theta_{W}}\left|D_{Z}(\hat{s})\right|^{2}\left(L_{q}^{2}+R_{q}^{2}\right) O_{Z}^{i j} O_{Z}^{i j \star}\left[\left(m_{\widetilde{\chi}_{i}^{0}}^{2}-\hat{u}\right)\left(m_{\widetilde{\chi}_{j}^{0}}^{2}-\hat{u}\right)+\right. \\
& M_{\hat{t} \hat{t}}=\frac{\left.\left(m_{\widetilde{\chi}_{i}^{0}}^{2}-\hat{t}\right)\left(m_{\widetilde{\chi}_{j}^{0}}^{2}-\hat{t}\right)-m_{\widetilde{\chi}_{i}^{0}} m_{\widetilde{\chi}_{j}^{0}} \hat{s}\left(O_{Z}^{i j 2}+O_{Z}^{i j \star 2}\right)\right],}{\left(\hat{t}-m_{\widetilde{q}_{k}}^{2}\right)\left(\hat{t}-m_{\widetilde{q}_{l}}^{2}\right)}\left(a_{i}^{L}\left(\widetilde{q}_{k}\right) a_{i}^{L \star}\left(\widetilde{q}_{l}\right)+a_{i}^{R}\left(\widetilde{q}_{k}\right) a_{i}^{R \star}\left(\widetilde{q}_{l}\right)\right)\left(a_{j}^{L}\left(\widetilde{q}_{k}\right) a_{j}^{L \star}\left(\widetilde{q}_{l}\right)+\right. \\
& M_{\hat{u} \hat{u}}=\frac{1}{\left(\hat{u}-m_{\widetilde{q}_{k}}^{2}\right)\left(\hat{u}-m_{\widetilde{q}_{l}}^{2}\right)}\left(a_{i}^{L \star}\left(\widetilde{q}_{k}\right) a_{i}^{L}\left(\widetilde{q}_{l}\right)+a_{i}^{R \star}\left(\widetilde{q}_{k}\right) a_{i}^{R}\left(\widetilde{q}_{l}\right)\right)\left(a_{j}^{L}\left(\widetilde{q}_{l}\right) a_{j}^{L \star}\left(\widetilde{q}_{k}\right)+\right. \\
& M_{\hat{t} \hat{u}}^{2}=\frac{(3.15)\left(m_{\widetilde{\chi}_{i}^{0}}^{2}-\hat{t}\right),}{\left(\hat{t}-m_{\widetilde{q}_{k}^{0}}^{2}\right)\left(\hat{u}-m_{\widetilde{q}_{l}}^{2}\right)}\left\{\frac { 1 } { 2 } \left[a_{i}^{L \star}\left(\widetilde{q}_{k}\right) a_{j}^{L}\left(\widetilde{q}_{l}\right) a_{j}^{R}\left(\widetilde{q}_{k}\right) a_{i}^{R \star}\left(\widetilde{q}_{l}\right)+a_{i}^{R \star}\left(\widetilde{q}_{k}\right) a_{j}^{R}\left(\widetilde{q}_{l}\right)\right.\right. \\
& 1
\end{aligned}
$$




$$
\begin{gathered}
\left.a_{i}^{L \star}\left(\widetilde{q}_{l}\right) a_{j}^{L}\left(\widetilde{q}_{k}\right)\right]\left(\left(m_{\widetilde{\chi}_{j}^{0}}^{2}-\hat{u}\right)\left(m_{\widetilde{\chi}_{i}^{0}}^{2}-\hat{u}\right)+\left(m_{\widetilde{\chi}_{j}^{0}}^{2}-\hat{t}\right)\left(m_{\widetilde{\chi}_{i}^{0}}^{2}-\hat{t}\right)-\hat{s}\left(\hat{s}-m_{\widetilde{\chi}_{i}^{0}}^{2}-m_{\widetilde{\chi}_{j}^{0}}^{2}\right)\right)+m_{\widetilde{\chi}_{i}^{0}}^{2} m_{\widetilde{\chi}_{j}^{0}}^{2} \\
\left.\times \hat{s}\left[a_{j}^{L \star}\left(\widetilde{q}_{l}\right) a_{i}^{L}\left(\widetilde{q}_{k}\right) a_{i}^{L}\left(\widetilde{q}_{l}\right) a_{j}^{L \star}\left(\widetilde{q}_{k}\right)+a_{j}^{R \star}\left(\widetilde{q}_{l}\right) a_{i}^{R}\left(\widetilde{q}_{k}\right) a_{i}^{R}\left(\widetilde{q}_{l}\right) a_{j}^{R \star}\left(\widetilde{q}_{k}\right)\right]\right\} \quad(3.17) \\
M_{\hat{s} \hat{u}}=\frac{e^{2}}{2 \sin ^{2} \Theta_{W} \cos ^{2} \Theta_{W}\left(\hat{u}-m_{\widetilde{q}_{k}}^{2}\right)}\left\{( R e [ D _ { Z } ( \hat { s } ) ] ) \left[L_{q} a_{i}^{L \star}\left(\widetilde{q}_{k}\right) a_{j}^{L}\left(\widetilde{q}_{k}\right) O_{Z}^{i j \star}-\right.\right. \\
\left.R_{q} a_{i}^{R \star}\left(\widetilde{q}_{k}\right) a_{j}^{R}\left(\widetilde{q}_{k}\right) O_{Z}^{i j}\right]\left(m_{\widetilde{\chi}_{i}^{0}}^{2}-\hat{u}\right)\left(m_{\widetilde{\chi}_{j}^{0}}^{2}-\hat{u}\right)+\left[R_{q} a_{i}^{R \star}\left(\widetilde{q}_{k}\right) a_{j}^{R}\left(\widetilde{q}_{k}\right) O_{Z}^{i j \star}-\right. \\
M_{\hat{s} \hat{t}}=\frac{\left.\left.e_{q} a_{i}^{L \star}\left(\widetilde{q}_{k}\right) a_{j}^{L}\left(\widetilde{q}_{k}\right) O_{Z}^{i j}\right] m_{\widetilde{\chi}_{i}^{0}} m_{\widetilde{\chi}_{j}^{0} \hat{s}} \hat{2}\right\}}{2 \sin ^{2} \Theta_{W} \cos ^{2} \Theta_{W}\left(\hat{t}-m_{\widetilde{q}_{k}}^{2}\right)}\left\{( R e [ D _ { Z } ( \hat { s } ) ] ) \left[R_{q} a_{j}^{R \star}\left(\widetilde{q}_{k}\right) a_{i}^{R}\left(\widetilde{q}_{k}\right) O_{Z}^{i j \star}-\right.\right. \\
\left.L_{q} a_{j}^{L \star}\left(\widetilde{q}_{k}\right) a_{i}^{L}\left(\widetilde{q}_{k}\right) O_{Z}^{i j}\right]\left(m_{\widetilde{\chi}_{i}^{0}}^{2}-\hat{t}\right)\left(m_{\widetilde{\chi}_{j}^{0}}^{2}-\hat{t}\right)+\left[L_{q} a_{j}^{L \star}\left(\widetilde{q}_{k}\right) a_{i}^{L}\left(\widetilde{q}_{k}\right) O_{Z}^{i j \star}-\right. \\
\left.\left.R_{q} a_{j}^{R \star}\left(\widetilde{q}_{k}\right) a_{i}^{R}\left(\widetilde{q}_{k}\right) O_{Z}^{i j}\right] m_{\widetilde{\chi}_{i}^{0}} m_{\widetilde{\chi}_{j}^{0} \hat{s}}\right\}
\end{gathered}
$$

The following abbreviation has been used

$$
D_{Z}\left(\hat{s}^{2}\right)=\frac{1}{\hat{s}^{2}-m_{Z}^{2}+i m_{Z} \Gamma_{Z}} .
$$

For calculation, we assume $m_{Z}=91.1887 \mathrm{GeV}$ and the widths of the gauge boson by $\Gamma_{Z}=2.499947 G e V$. The basic parton model expression for the hadron-hadron collision $h_{1}\left(p_{1}\right) h_{2}\left(p_{2}\right) \rightarrow \widetilde{\chi}_{i}^{0}\left(k_{i}\right) \widetilde{\chi}_{j}^{0}\left(k_{j}\right),[14,15]$ is

$$
\begin{gathered}
d \sigma\left(h_{1}\left(p_{1}\right) h_{2}\left(p_{2}\right) \rightarrow \widetilde{\chi}_{i}^{0}\left(k_{i}\right) \widetilde{\chi}_{j}^{0}\left(k_{j}\right)\right)=\sum \iint d x_{1} d x_{2} G_{q_{1} / h_{1}}\left(x_{1}, Q\right) G_{q_{2} / h_{2}}\left(x_{2}, Q\right) \\
d \sigma\left(q_{1} q_{2} \rightarrow \widetilde{\chi}_{i}^{0} \widetilde{\chi}_{j}^{0}\right) \frac{1}{1+\delta_{q_{1} q_{2}}}
\end{gathered}
$$

with $\widetilde{\chi}_{i}^{0}, \widetilde{\chi}_{J}^{0}$ being the two produced massive particles of mass $m_{\widetilde{\chi}_{i}^{0}}, m_{\widetilde{\chi}_{j}^{0}}$. Here $G_{q_{1} / h_{1}}\left(x_{1}, Q\right)$ is the distribution function of partons of type $\left(q_{1}=q, \bar{q}\right)$, in the 
hadron of type $h_{1}$ at a factorization scale Q. Taking the $h_{1} h_{2}$-c.m. system as the lab-system, the lab-momenta of the produced $\widetilde{\chi}_{i}^{0}$ and $\widetilde{\chi}_{j}^{0}$ are $[16]$

$$
k_{i}^{\mu}=\left(E_{i}, k_{T}, k_{i} \cos \theta\right), \quad k_{j}^{\mu}=\left(E_{j},-k_{T}, k_{j} \cos \theta\right),
$$

where their transverse momenta are obviously just opposite

$$
k_{T}=k_{T_{i}}=-k_{T_{j}},
$$

while their transverse energies $E_{T_{i}}=\sqrt{k_{T}^{2}+m_{\widetilde{\chi}_{i}^{0}}^{2}}, E_{T_{j}}=\sqrt{k_{T}^{2}+m_{\widetilde{\chi}_{j}^{0}}^{2}}$ are used to define

$$
\begin{gathered}
x_{T_{i}}=\frac{2 E_{T_{i}}}{\sqrt{s}}, \beta_{T_{i}}=k_{T} / E_{T_{i}}=\sqrt{1-\frac{4 m_{\widetilde{\chi}_{i}^{0}}^{2}}{s x_{T_{i}}^{2}}}, \\
x_{T_{j}}=\frac{2 E_{T_{j}}}{\sqrt{s}}, \beta_{T_{j}}=k_{T} / E_{T_{j}}=\sqrt{1-\frac{4 m_{\widetilde{\chi}_{j}^{0}}^{2}}{s x_{T_{j}}^{2}}},
\end{gathered}
$$

Note that

$$
E_{T_{j}}^{2}=E_{T_{i}}^{2}+m_{\widetilde{\chi}_{j}^{0}}^{2}-m_{\widetilde{\chi}_{i}^{0}}^{2}, \quad x_{T_{j}}^{2}=x_{T_{i}}^{2}+4 \cdot \frac{\left(m_{\widetilde{\chi}_{j}^{0}}^{2}-m_{\widetilde{\chi}_{i}^{0}}^{2}\right)}{s}
$$

The rapidites and production angles of $\widetilde{\chi}_{i}^{0}, \widetilde{\chi}_{j}^{0}$, in the lab-system, are related to their energies and momenta along the beam-axis of hadron $h_{1}$, by

$$
y_{i}=\frac{1}{2} \ln \frac{E_{i}+k_{i} \cos \theta_{i}}{E_{i}-k_{i} \cos \theta_{i}}, \quad y_{j}=\frac{1}{2} \ln \frac{E_{j}+k_{j} \cos \theta_{j}}{E_{j}-k_{j} \cos \theta_{j}}
$$

The center-of-mass rapidity $\bar{y}$ of the $\widetilde{\chi}_{i}^{0} \widetilde{\chi}_{j}^{0}$ pair, and their respective rapidities $y_{i}^{\star}$ in their own c.m. frame, are defined as

$$
\begin{gathered}
y_{i}=\bar{y}+y_{i}^{\star}, \quad y_{j}=\bar{y}+y_{j}^{\star}, \\
\Delta \equiv y_{i}-y_{j}=y_{i}^{\star}-y_{j}^{\star} .
\end{gathered}
$$


The fractional momenta of the incoming partons are expressed in the terms of their lab-momenta by

$$
\begin{gathered}
p_{1}=\frac{s}{2}\left(x_{1}, 0,0, x_{1}\right), \quad p_{2}=\frac{s}{2}\left(x_{2}, 0,0,-x_{2}\right), p=p_{1}+p_{2}, \\
p^{0}=\frac{\sqrt{s}}{2}\left(x_{1}+x_{2}\right)=E_{i}+E_{j}, \quad p_{3}=\frac{\sqrt{s}}{2}\left(x_{1}-x_{2}\right)=\left(k_{i} \cos \theta_{i}+k_{j} \cos \theta_{j}\right),
\end{gathered}
$$

which lead to

$$
\begin{gathered}
x_{1}=\frac{1}{2}\left[x_{T_{i}} e^{y_{i}}+x_{T_{j}} e^{y_{j}}\right]=\frac{M}{\sqrt{s}} e^{\bar{y}}, \\
x_{2}=\frac{1}{2}\left[x_{T_{i}} e^{-y_{i}}+x_{T_{j}} e^{-y_{j}}\right]=\frac{M}{\sqrt{s}} e^{-\bar{y}}, \\
\hat{s}=M^{2}=\left(p_{1}+p_{2}\right)^{2}=x_{1} x_{2} s=\frac{s}{4}\left[x_{T_{i}}^{2}+x_{T_{j}}^{2}+2 x_{T_{i}} x_{T_{j}} \cosh (\Delta y)\right] .
\end{gathered}
$$

Using this, $\hat{s}, x_{1}, x_{2}$ may be calculated in terms of the final particle rapidities $y_{i}, y_{j}$ and their transverse momenta. From them, $\bar{y}$ is also obtained through $\left(y_{i}^{\star}, y_{j}^{\star}\right)$. The remaining Mandelstam invariants of the subprocesses satisfy

$$
\begin{gathered}
\hat{t}=\left(p_{1}-k_{i}\right)^{2}=m_{\widetilde{\chi}_{i}^{0}}^{2}-M\left(E_{i}^{\star}-k^{\star} \cos \theta^{\star}\right)=m_{\widetilde{\chi}_{i}^{0}}^{2}-\frac{x_{T_{i}}}{2} M \sqrt{s} e^{-y_{i}^{\star}}= \\
m_{\widetilde{\chi}_{i}^{0}}^{2}-\frac{s}{2} x_{1} x_{T_{i}} e^{-y_{i}}=m_{\widetilde{\chi}_{j}^{0}}^{2}-M\left(E_{j}^{\star}-k^{\star} \cos \theta^{\star}\right)= \\
m_{\widetilde{\chi}_{j}^{0}}^{2}-\frac{x_{T_{i}}}{2} M \sqrt{s} e^{y_{j}^{\star}}=m_{\widetilde{\chi}_{j}^{0}}^{2}-\frac{s}{2} x_{2} x_{T_{j}} e^{y_{j}} \\
\hat{u}=\left(p_{1}-k_{j}\right)^{2}=m_{\widetilde{\chi}_{i}^{0}}^{2}-M\left(E_{i}^{\star}+k^{\star} \cos \theta^{\star}\right)=m_{\widetilde{\chi}_{i}^{0}}^{2}-\frac{x_{T_{i}}}{2} M \sqrt{s} e^{y_{i}^{\star}}= \\
m_{\widetilde{\chi}_{j}}^{2}-\frac{s}{2} x_{2} x_{T_{i}} e^{y_{i}}=m_{\widetilde{\chi}_{j}^{0}}^{2}-M\left(E_{j}^{\star}+k^{\star} \cos \theta^{\star}\right)=
\end{gathered}
$$




$$
\begin{gathered}
m_{\widetilde{\chi}_{j}^{0}}^{2}-\frac{x_{T_{j}}}{2} M \sqrt{s} e^{-y_{j}^{\star}}=m_{\widetilde{\chi}_{j}^{0}}^{2}-\frac{s}{2} x_{1} x_{T_{j}} e^{-y_{j}} . \\
\tau=\frac{\hat{s}}{s}=x_{1} x_{2},
\end{gathered}
$$

where $\theta^{\star}$ describes $\widetilde{\chi}_{i}^{0}$ production angle in $\widetilde{\chi}_{i}^{0} \widetilde{\chi}_{j}^{0}$-c.m. frame (the $\widetilde{\chi}_{j}^{0}$ one being $\left.\pi-\theta^{\star}\right)$. The energies of the two final particles in their c.m.-frame are

$$
E_{i}^{\star}=\frac{\hat{s}+m_{\widetilde{\chi}_{i}^{0}}^{2}-m_{\widetilde{\chi}_{j}^{0}}^{2}}{2 \sqrt{\hat{s}}}, E_{j}^{\star}=\frac{\hat{s}+m_{\widetilde{\chi}_{j}^{0}}^{2}-m_{\widetilde{\chi}_{i}^{0}}^{2}}{2 \sqrt{\hat{s}}},
$$

their momentum is

$$
p^{\star}=\frac{1}{2 M}\left[\left(M^{2}-m_{\widetilde{\chi}_{i}^{0}}^{2}-m_{\widetilde{\chi}_{j}^{0}}^{2}\right)^{2}-4 m_{\widetilde{\chi}_{i}^{0}}^{2} m_{\widetilde{\chi}_{j}^{0}}^{2}\right]^{0.5}
$$

and their velocities

$$
\begin{aligned}
& \beta_{i}^{\star}=k^{\star} / E_{i}^{\star}=\frac{\left[\left(M^{2}-m_{\widetilde{\chi}_{i}^{0}}^{2}-m_{\widetilde{\chi}_{j}^{0}}^{2}\right)^{2}-4 m_{\widetilde{\chi}_{i}^{0}}^{2} m_{\widetilde{\chi}_{j}^{0}}^{2}\right]^{1 / 2}}{M^{2}+\left(m_{\tilde{\chi}_{i}}-m_{\widetilde{\chi}_{j}}\right)^{2}}, \\
& \beta_{j}^{\star}=k^{\star} / E_{j}^{\star}=\frac{\left[\left(M^{2}-m_{\widetilde{\chi}_{i}^{0}}^{2}-m_{\widetilde{\chi}_{j}^{0}}^{2}\right)^{2}-4 m_{\widetilde{\chi}_{i}^{0}}^{2} m_{\widetilde{\chi}_{j}^{0}}^{2}\right]^{1 / 2}}{M^{2}-\left(m_{\tilde{\chi}_{i}}-m_{\widetilde{\chi}_{j}}\right)^{2}},
\end{aligned}
$$

We also have

$$
\begin{gathered}
\cos \theta^{\star}=\frac{\tan y_{i}^{\star}}{\beta_{i}^{\star}}=-\frac{\tan y_{j}^{\star}}{\beta_{j}^{\star}}, \\
\sin \theta^{\star}=\frac{p_{T}}{p^{\star}}, \\
\chi_{i}=e^{2 y_{i}^{\star}}=\frac{\hat{u}-m_{\widetilde{\chi}_{i}}^{2}}{\hat{t}-m_{\widetilde{\chi}_{i}}^{2}}=\frac{1+\beta_{i}^{\star} \cos \theta^{\star}}{1-\beta_{i}^{\star} \cos \theta^{\star}}, \\
\chi_{j}=e^{2 y_{j}^{\star}}=\frac{\hat{t}-m_{\widetilde{\chi}_{j}}^{2}}{\hat{u}-m_{\tilde{\chi}_{j}}^{2}}=\frac{1-\beta_{j}^{\star} \cos \theta^{\star}}{1+\beta_{j}^{\star} \cos \theta^{\star}},
\end{gathered}
$$




$$
\begin{aligned}
& \beta_{i}^{\star} \cos \theta^{\star}=\frac{\hat{u}-\hat{t}}{\hat{u}+\hat{t}}=\frac{\chi_{i}-1}{\chi_{i}+1}, \\
& \chi_{j}=\frac{\chi_{i}\left(m_{\tilde{\chi}_{j}}^{2}-m_{\tilde{\chi}_{i}}^{2}\right)+M^{2}}{\chi_{i} M^{2}+m_{\tilde{\chi}_{j}}^{2}-m_{\tilde{\chi}_{i}}^{2}}, \\
& E_{T_{i}}=\frac{E_{i}^{\star}}{\cosh y_{i}^{\star}}, \\
& k_{T}^{2}=\frac{\left(M^{2}+m_{\widetilde{\chi}_{i}^{0}}^{2}-m_{\widetilde{\chi}_{j}^{0}}^{2}\right)^{2} \chi_{i}-M^{2} m_{\widetilde{\chi}_{i}^{0}}^{2}\left(1+\chi_{i}\right)^{2}}{M^{2}\left(1+\chi_{i}\right)^{2}}, \\
& x_{T_{i}}^{2}=\frac{4\left(M^{2}+m_{\chi_{i}^{0}}^{2}-m_{\chi_{j}^{0}}\right)^{2} \chi_{i}}{M^{2} s\left(1+\chi_{i}\right)^{2}}, \\
& x_{T_{j}}^{2}=\frac{4\left(M^{2}+m_{\chi_{j}}^{2}-m_{\chi_{i}}\right)^{2} \chi_{j}}{M^{2} s\left(1+\chi_{j}\right)^{2}} .
\end{aligned}
$$

Using (3.20) we define the expression for the cross section in terms of the overall center-of-mass rapidities of the two jets yielding

$$
\frac{d \sigma}{d y_{i} d y_{j} d k_{T}^{2}}=x_{1} x_{2} \sum_{q} G_{q_{1} / h_{1}}\left(x_{1}, Q\right) G_{q_{2} / h_{2}}\left(x_{2}, Q\right) \frac{d \sigma}{d \hat{t}}\left(q_{1} q_{2} \rightarrow \widetilde{\chi}_{i}^{0} \widetilde{\chi}_{j}^{0}\right) .
$$

\section{NUMERICAL RESULTS AND DISCUSSION}

In this section, we discuss the numerical results for the process $p p \rightarrow \widetilde{\chi}_{i}^{0} \widetilde{\chi}_{j}^{0}$ at LHC (c.m energy $\sqrt{s}=14 \mathrm{TeV}$ ) generated by the subprocesses $q \bar{q}$. As we assume, $\widetilde{\chi}_{1}^{0}$ is likely to be the LSP, the three types of channels: $q \bar{q} \rightarrow \widetilde{\chi}_{1}^{0} \widetilde{\chi}_{1}^{0}$, $q \bar{q} \rightarrow \widetilde{\chi}_{2}^{0} \widetilde{\chi}_{2}^{0}, q \bar{q} \rightarrow \widetilde{\chi}_{1}^{0} \widetilde{\chi}_{2}^{0}$, would be the most dominant neutralino pair production processes, which may lead to the first detection of SUSY particles at the LHC. The numerical results of the cross sections of these three processes 
have been presented and their dependencies on the basic SUSY parameters have been discussed. We divide the input MSSM parameters into two parts. One part is for the general parameters included also in SM, and the other part is the ino and squark sectors of MSSM. For the first parameter part, we take $m_{Z^{0}}=91.1887 \mathrm{GeV}, \sin \Theta_{W}^{2}=0.2315, \alpha_{E}=1 / 137$. For the second part, we just limit the values of $M_{1}, M_{2}$ and $\mu$ to be real, positive and below $1 \mathrm{TeV}$, and take $\tan \beta=2, m_{\widetilde{u}_{1}}=m_{\widetilde{d}_{1}}=300 \mathrm{GeV}, m_{\widetilde{u}_{2}}=m_{\widetilde{d}_{2}}=500 \mathrm{GeV}$. Also, we fix the heavy chargino mass as $m_{\tilde{\chi}_{2}^{+}}=450 \mathrm{GeV}$ and for the lightest chargino mass $m_{\widetilde{\chi}_{1}^{+}}=150 G e V$. By using Eqs. $(2.7,2.8)$ with above chargino mass values, one may have two choices of parameter sets for $\mu$ and $M_{2}$ in two extreme cases, which are the Higgsino-like and the gaugino-like respectively. For the Higgsino-like case, we get $M_{2}=437.96 \mathrm{GeV}, \mu=169.753 \mathrm{GeV}$, $M_{1}=219.703 \mathrm{GeV}$ and by inserting the values of $M_{2}, \mu$ and $M_{1}$ into Eq.(2.11) for neutralino masses, we get

$m_{\widetilde{\chi}_{1}^{0}}=123.242 \mathrm{GeV}, m_{\widetilde{\chi}_{2}^{0}}=179.475 \mathrm{GeV}, m_{\widetilde{\chi}_{3}^{0}}=238.199 \mathrm{GeV}, m_{\widetilde{\chi}_{4}^{0}}=458.479 \mathrm{GeV}$

For the gaugino-like case, we have $M_{2}=169.753 \mathrm{GeV}, \mu=437.96 \mathrm{GeV}$, $M_{1}=85.157 \mathrm{GeV}$ and by inserting the values of $M_{2}, \mu$ and $M_{1}$ into Eq.(2.11) for neutralino masses, we then get

$m_{\widetilde{\chi}_{1}^{0}}=77.212 \mathrm{GeV}, m_{\widetilde{\chi}_{2}^{0}}=153.859 \mathrm{GeV}, m_{\widetilde{\chi}_{3}^{0}}=449.420 \mathrm{GeV}, m_{\widetilde{\chi}_{4}^{0}}=452.443 \mathrm{GeV}$

For full discussion, we present the results for the mixture case as well. We take the heavy chargino mass $m_{\widetilde{\chi}_{2}^{+}}=280.6 \mathrm{GeV}$ and for the lightest chargino mass $m_{\widetilde{\chi}_{1}^{+}}=128 \mathrm{GeV}$, the corresponding outputs obtained as $M_{2}=\mu=$ $200.598 \mathrm{GeV}, M_{1}=100.63 \mathrm{GeV}$ and also by inserting the values of $M_{2}, \mu$ and 
$M_{1}$ into Eq.(2.11) for the neutralino masses, we get

$m_{\tilde{\chi}_{1}^{0}}=72.576 \mathrm{GeV}, m_{\tilde{\chi}_{2}^{0}}=147.127 \mathrm{GeV}, m_{\tilde{\chi}_{3}^{0}}=207.184 \mathrm{GeV}, m_{\tilde{\chi}_{4}^{0}}=278.635 \mathrm{GeV}$

As an example for the quark distribution function inside the proton, we use the MRST2003c package[17]. After this, the cross sections of the subprocesses $q \bar{q} \rightarrow \widetilde{\chi}_{1}^{0} \widetilde{\chi}_{1}^{0}, \widetilde{\chi}_{1}^{0} \widetilde{\chi}_{2}^{0}, \widetilde{\chi}_{2}^{0} \widetilde{\chi}_{2}^{0}$ can be numerically evaluated. For illustration, we have calculated the total cross sections of the subprocesses $u \bar{u} \rightarrow \widetilde{\chi}_{1}^{0} \widetilde{\chi}_{1}^{0}$, $\widetilde{\chi}_{1}^{0} \widetilde{\chi}_{2}^{0}, \widetilde{\chi}_{2}^{0} \widetilde{\chi}_{2}^{0}$, and $d \bar{d} \rightarrow \widetilde{\chi}_{1}^{0} \widetilde{\chi}_{1}^{0}, \widetilde{\chi}_{1}^{0} \widetilde{\chi}_{2}^{0}, \widetilde{\chi}_{2}^{0} \widetilde{\chi}_{2}^{0}$, the dependence on the beam energies, on the mass of squarks and also of the $M_{2}$ gaugino mass. We will illustrate this for three extremely different scenarios. In Fig.2-4, we show the dependence of the total cross sections for the subprocesses $u \bar{u} \rightarrow \widetilde{\chi}_{1}^{0} \widetilde{\chi}_{1}^{0}$, $\widetilde{\chi}_{1}^{0} \widetilde{\chi}_{2}^{0}, \widetilde{\chi}_{2}^{0} \widetilde{\chi}_{2}^{0}$ of the beam energy. In our calculations, the beam energy for the subprocesses $\hat{s}$ changes in the region $(400 \div 5000) \mathrm{GeV}$, which corresponds to the beam energy for process $s(500 \div 16000) \mathrm{GeV}$. As shown in Fig.2-4, all subprocesses in the gaugino-like scenario, the total cross section is 30 percent larger than the mixing scenario in magnitude, and larger than in the Higgsino-like scenario as 1.79-2 order of magnitude. As seen from Figs.2-4, in all three scenarios, the dependencies of the cross section on the beam energy demonstrate the same behavior. Also, as seen from Figs.2-4, all three scenarios, by increasing the beam energy from $400 \mathrm{GeV}$ to $3600 \mathrm{GeV}$, the total cross section is monotonically increasing. In Figs.5-7, we show the dependence of the total cross sections for the subprocesses $d \bar{d} \rightarrow \widetilde{\chi}_{1}^{0} \widetilde{\chi}_{1}^{0}, \widetilde{\chi}_{1}^{0} \widetilde{\chi}_{2}^{0}, \widetilde{\chi}_{2}^{0} \widetilde{\chi}_{2}^{0}$ of the beam energy. As shown in Fig.5-6, all subprocesses in the gaugino-like scenario, the total cross section is 8 percent larger than the mixing scenario in magnitude, and larger than in the Higgsino like scenario as 2-2.137 order 
of magnitude. As shown in Fig.7, in the gaugino-like scenario, the total cross section is almost 2 percent larger than the mixing scenario in magnitude, and larger than in the Higgsino-like scenario as two order of magnitude. As seen from Figs.5-7, in all three scenarios, the dependence of the cross section on the beam energy demonstrates the same behavior. With the increase of the beam energy from $400 \mathrm{GeV}$ to $4000 \mathrm{GeV}$, the total cross section is monotonically increasing. The total cross section of the subprocesses $u \bar{u} \rightarrow \widetilde{\chi}_{1}^{0} \widetilde{\chi}_{2}^{0}$ in the gaugino-like scenario, appears in the range of 2 to $140 \mathrm{fb}$ and should be observable at LHC. It should be noted that, one of the subprocesses, $q \bar{q} \rightarrow \widetilde{\chi}_{1}^{0} \widetilde{\chi}_{2}^{0}$ dominates all three scenarios. According to our opinion, it may be used as a probe for an experimental search on the neutralino pair. Figs.8-13 show the dependence of the total cross sections for the subprocesses $u \bar{u} \rightarrow \widetilde{\chi}_{1}^{0} \widetilde{\chi}_{1}^{0}$, $\widetilde{\chi}_{1}^{0} \widetilde{\chi}_{2}^{0}, \widetilde{\chi}_{2}^{0} \widetilde{\chi}_{2}^{0}$, and $d \bar{d} \rightarrow \widetilde{\chi}_{1}^{0} \widetilde{\chi}_{1}^{0}, \widetilde{\chi}_{1}^{0} \widetilde{\chi}_{2}^{0}, \widetilde{\chi}_{2}^{0} \widetilde{\chi}_{2}^{0}$, of the $M_{2}$. As seen from Figs.810, the cross section is decreasing when the $M_{2}$ gaugino mass is increasing. But, as seen from Fig.11, the dependence of the total cross sections for the subprocesses $d \bar{d} \rightarrow \widetilde{\chi}_{1}^{0} \widetilde{\chi}_{1}^{0}, \widetilde{\chi}_{1}^{0} \widetilde{\chi}_{2}^{0}, \widetilde{\chi}_{2}^{0} \widetilde{\chi}_{2}^{0}$ of the $M_{2}$ have another character. As shown in Figs.12-13, the cross section is decreasing with increasing $M_{2}$ in the range of 300 to 400 and it has a minimum approximately at one point $M_{2}=400 \mathrm{GeV}$. After this point, the cross section increases with $M_{2}$ gaugino mass increasing. Behavior of the cross section is decreasing when the $M_{2}$ gaugino mass is increasing as expected. Because, we have obtained the following relation for the dependence cross section depending on the beam energy: $\sigma($ Higgsino - like $)<\sigma($ Mixture - case $)<\sigma($ Gaugino - like $)$. This relation corresponds to $M_{2}($ Gaugino - like $)<M_{2}($ Mixture - case $)<$ $M_{2}($ Higgsino - like), respectively. Therefore, the cross section is decreasing 
when the $M_{2}$ gaugino mass is increasing. Figs.14-19 show the dependence of the total cross sections for the subprocesses $u \bar{u} \rightarrow \widetilde{\chi}_{1}^{0} \widetilde{\chi}_{1}^{0}, \widetilde{\chi}_{1}^{0} \widetilde{\chi}_{2}^{0}, \widetilde{\chi}_{2}^{0} \widetilde{\chi}_{2}^{0}$, and $d \bar{d} \rightarrow \widetilde{\chi}_{1}^{0} \widetilde{\chi}_{1}^{0}, \widetilde{\chi}_{1}^{0} \widetilde{\chi}_{2}^{0}, \widetilde{\chi}_{2}^{0} \widetilde{\chi}_{2}^{0}$, of the squarks mass. As shown in Figs.14-19, in all subprocesses and scenarios, the cross section monotonically decreases with an increase in the squark masses. Therefore, an experimental search for the neutralino pair at lower values of the squark mass is preferable. In Figs.2022 , we show the dependence of the differential cross sections for the process $p p \rightarrow \widetilde{\chi}_{i}^{0} \widetilde{\chi}_{j}^{0}$ as a function of the $k_{T}$ transverse momentum of the neutralino pair at rapidity $y_{i}=y_{j}=0$. As seen from Figs.20-22, the differential cross sections decrease monotonically pursuant to an increase in the $k_{T}$ transverse momentum of the neutralino pair. It should be noted that, one of the process $p p \rightarrow \widetilde{\chi}_{1}^{0} \widetilde{\chi}_{2}^{0}$ dominates all three scenarios. As mentioned in above, that our results disagree with the results in [6]. So, the expression for the cross sections in Ref.[6] is wrong. Therefore, our numerical results also disagree with the numerical results in [6]. We compared our results with [18]. The pair production rate of $\widetilde{\chi}_{1}^{0} \widetilde{\chi}_{2}^{0}$ via gluon-gluon fusion is about few ten percent of that via quark-antiquark annihilation at the LHC. The cross section of $\widetilde{\chi}_{2}^{0} \widetilde{\chi}_{2}^{0}$ via gluon-gluon fusion is about few percent of that via quark-antiquark annihilation at the LHC.

\section{CONCLUSION}

In this paper, we have considered the neutralino pair production processes in proton-proton collisions at LHC (c.m energy $\sqrt{s}=14 T e V)$. In the description, we have taken into account the subprocess $q\left(p_{1}\right) \bar{q}\left(p_{2}\right) \rightarrow \widetilde{\chi}_{i}^{0}\left(k_{1}\right) \widetilde{\chi}_{j}^{0}\left(k_{2}\right)$. We have given detail illustrations for the center-of-mass energy, squarks and 
$M_{2}$ gaugino masses for three extremely different scenarios. For illustration, we have calculated the total cross sections of the subprocesses $q \bar{q} \rightarrow \widetilde{\chi}_{1}^{0} \widetilde{\chi}_{1}^{0}$, $\widetilde{\chi}_{1}^{0} \widetilde{\chi}_{2}^{0}, \widetilde{\chi}_{2}^{0} \widetilde{\chi}_{2}^{0}$, and the dependence on the beam energies, on the mass of squarks and also of the $M_{2}$ gaugino mass. We have illustrated this for three extremely different scenarios. Fig.2-7 show the dependence of the total cross sections for the subprocesses $q \bar{q} \rightarrow \widetilde{\chi}_{1}^{0} \widetilde{\chi}_{1}^{0}, \widetilde{\chi}_{1}^{0} \widetilde{\chi}_{2}^{0}, \widetilde{\chi}_{2}^{0} \widetilde{\chi}_{2}^{0}$ of the beam energy. As seen from Figs.2-7, in all three scenarios, the dependencies of the cross section on the beam energy demonstrate the same behavior. The total cross section of the subprocesses $u \bar{u} \rightarrow \widetilde{\chi}_{1}^{0} \widetilde{\chi}_{2}^{0}$ in the gaugino-like scenario, appears in the range of 2 to $140 \mathrm{fb}$ and should be observable at LHC. It should be noted that, one of the subprocesses, $u \bar{u} \rightarrow \widetilde{\chi}_{1}^{0} \widetilde{\chi}_{2}^{0}$ dominates all three scenarios. According to our opinion, it may be used as a probe for an experimental search on the neutralino pair. Figs.8-13 show the dependence of the total cross sections for the subprocesses $q \bar{q} \rightarrow \widetilde{\chi}_{1}^{0} \widetilde{\chi}_{1}^{0}, \widetilde{\chi}_{1}^{0} \widetilde{\chi}_{2}^{0}, \widetilde{\chi}_{2}^{0} \widetilde{\chi}_{2}^{0}$, of the $M_{2}$. Figs.14-19 show the dependence of the total cross sections for the subprocesses $q \bar{q} \rightarrow \widetilde{\chi}_{1}^{0} \widetilde{\chi}_{1}^{0}, \widetilde{\chi}_{1}^{0} \widetilde{\chi}_{2}^{0}$, $\widetilde{\chi}_{2}^{0} \widetilde{\chi}_{2}^{0}$ of the squarks mass. As shown in Figs.14-19, in all subprocesses and scenarios, the cross section monotonically decreases with an increase in squark masses. It should be underlined that for all three scenarios, the dependence of the total cross section of the subprocesses $q \bar{q} \rightarrow \widetilde{\chi}_{i}^{0} \widetilde{\chi}_{j}^{0}$ on the beam energy is dominated by one of the subprocesses, $q \bar{q} \rightarrow \widetilde{\chi}_{1}^{0} \widetilde{\chi}_{2}^{0}$ and the dependence of the total cross section of the subprocesses $q \bar{q} \rightarrow \widetilde{\chi}_{i}^{0} \widetilde{\chi}_{j}^{0}$ on the mass of squarks is dominated by one of the subprocesses, $q \bar{q} \rightarrow \widetilde{\chi}_{1}^{0} \widetilde{\chi}_{1}^{0}$. These findings may be used as a probe in an experimental search for the neutralino pair at LHC. In Figs.20-22, we have shown the dependence of the differential cross sections for the process $p p \rightarrow \widetilde{\chi}_{i}^{0} \widetilde{\chi}_{j}^{0}$ as a function of the $k_{T}$ transverse momentum of 
the neutralino pair at rapidity $y_{i}=y_{j}=0$. In this case, one of the process $p p \rightarrow \widetilde{\chi}_{1}^{0} \widetilde{\chi}_{2}^{0}$ dominate all three scenarios. These features make the neutralino pair production processes rather interesting for testing the SUSY dynamics at LHC. The reason is that they provide tests which will be complementary to those addressing the cascade decays of initially produced colored SUSY particles to eventually $\widetilde{\chi}_{1}^{0}$, which is here assumed to be the LSP; e.g. studies of mass spectra and decay branching ratios [19]. In particular, consistency checks should thus become available, allowing the strengthening of possible constraints on the validity of specific models. Moreover, in such neutralino pair production, the role of the Majorana nature of the final state particles is more prominent than in decays involving just one neutralino at a time. Since no such states have been observed in the past, it would be interesting to have eventually some experimental support of our understanding of the Majorana nature. These results imply an interesting complementarity between the future LHC measurements, the related $\gamma \gamma \rightarrow \widetilde{\chi}_{i}^{0} \widetilde{\chi}_{j}^{0}$ measurements at a future Linear Collider and the Dark Matter searches in cosmic experiments.

\section{Acknowledgments}

Two of authors, A. Ahmadov and I. Boztosun are grateful to TÜBİTAK Grant-2221 (BAYG) as well as TÜBİTAK Grant: TBAG-2398. One of us, A. Ahmadov is grateful also to NATO Reintegration Grant-980779 
[1] S.L. Glashow, Nucl. Phys. 22 (1961) 579; S. Weinberg, Phys. Rev. Lett. 19 (1967) 1264; A. Salam, Pros. $8^{\text {th }}$ Nobel Symposium Stockolm 1968, ed. N. Svartholm (Almquist and Wiksells, Stockolm 1968) p.367; H. D. Politzer, Phys. Rept. 14 (1974) 129.

[2] J. Ellis et al., Nucl. Phys. B238 (1984) 453.

[3] S. P. Martin, Phys. Rev. D46 (1992) 2769; E. Diehl, G. L. Kane, C. Kolda and J. D. Wells, Phys.Rev. D52 (1995) 4223.

[4] H. E. Haber, G. L. Kane, Phys. Rept. 117 (1985) 75.

[5] G. J. Gounaris, J. Layssac, P. I. Porfyriadis and F.M. Renard, Phys. Rev. D70 (2004) 03304.

[6] Han Liang, Ma Wen-Gan, Jiong Yi, Zhou Mian-Lai and Zhou Hong, Commun. Theoretical Phys. 34 (2000) 115.

[7] H. P. Nilles, Phys. Rept. 110 (1984) 1.

[8] M. Drees and S. P. Martin, hep-ph/9504324.

[9] V. Barger, M. S. Berger, P. Ohmann, R. Phillips, Phys. Lett. B314 (1993) 351; P. Langacker, N. Polonsky, Phys. Rev. D49 (1994) 1454; S. Kelley, J. L. Lopez, D. V. Nanopoulos, Phys. Lett. B274, (1992) 387.

[10] J. L. Kneur and G. Moultaka, Phys. Rev. D59 (1999) 015005.

[11] S. Ambrosanio and B. Mele, Phys. Rev. D52 (1995) 3900.

[12] G. Moultaka, "Extracting chargino/neutralino mass parameters from physical observables", Talk given at $29^{\text {th }}$ International Conference on High-Energy physics (ICHEP 98), Vancouver, Canada, 23-29 July 1998. In "Vancouver 1998, High Energy physics, vol.2" 1703-1709, hep-ph/9810214.

[13] J. Rosiek, Phys. Rev. D41 (1990) 3464; hep-ph/9411250.

[14] J. F. Owens, Rev. Mod. Phys. 59 (1987) 465.

[15] R. Kh. Muradov and A. I. Ahmadov, Central. Eur. J. Phys.3(3) (2005) 433.

[16] E. Bycling and K. Kajante, Particle Kinematics, Wiley, New York. 1973.

[17] MRST2003c.f can be obtained from http://durpdg.dur.ac.uk/hepdata/pdf.html. See also, A. D. Martin, R. G. Roberts, W. J. Stirling and R. S.Thorne, hep-ph/0307262; R. S.Thorne, hep-ph/0309343.

[18] Jiang Yi, Ma Wen-Gan, Han Liang, Yu Zeng-Hui and H. Pietschmann, Phys. Rev. D62, 
(2000) 035006.

[19] B. C. Allanach, G. A. Blair, S. Kraml, H. U. Martyn, G. Polesello, W. Porod and P. M. Zerwas, hep-ph/0403133; G. Weiglein, hep-ph/0404108. 


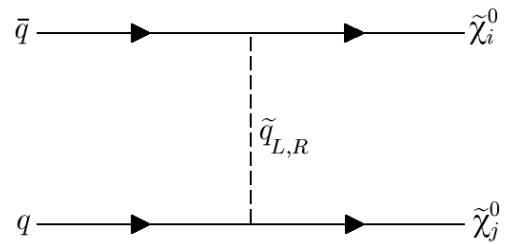

(a)

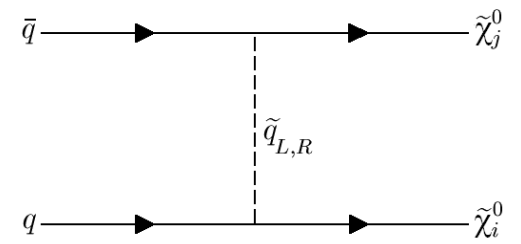

(b)

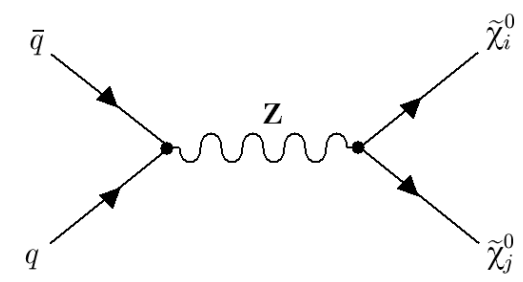

(c)

FIG. 1: Feynman diagrams for $q \bar{q} \rightarrow \widetilde{\chi}_{i}^{0} \widetilde{\chi}_{j}^{0}$ process. 


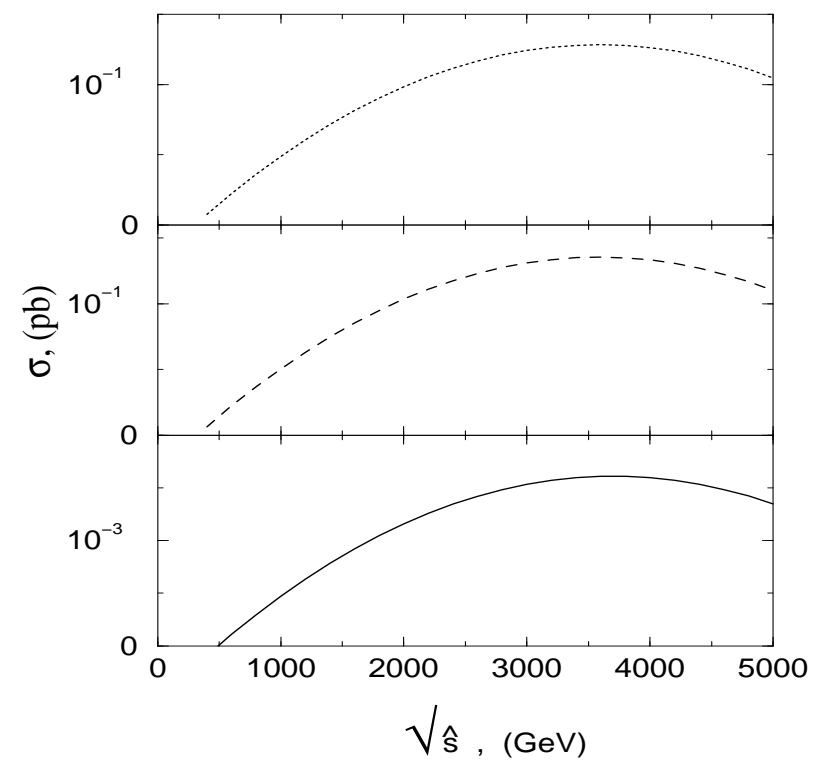

FIG. 2: The cross sections of the subprocess $u \bar{u} \rightarrow \widetilde{\chi}_{1}^{0} \widetilde{\chi}_{1}^{0}$ as a function of $\sqrt{\hat{s}}$. The curves correspond to: solid-Higgsino-like, dashed-gaugino-like and dotted-mixture cases, respectively.

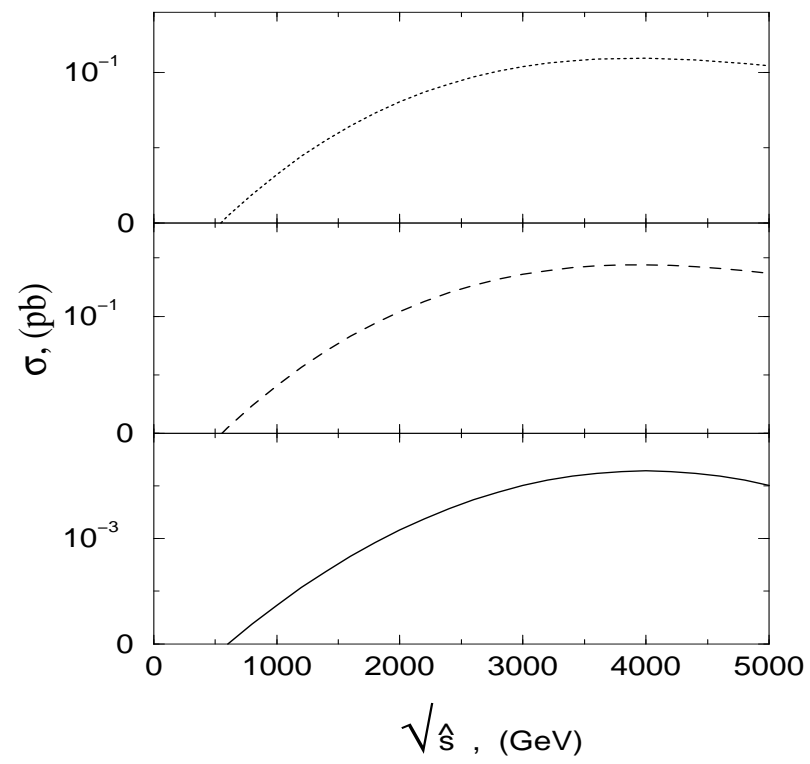

FIG. 3: The cross sections of the subprocess $u \bar{u} \rightarrow \widetilde{\chi}_{1}^{0} \widetilde{\chi}_{2}^{0}$ as a function of $\sqrt{\hat{s}}$. The curves correspond to: solid-Higgsino-like, dashed-gaugino-like and dotted-mixture cases, respectively. 


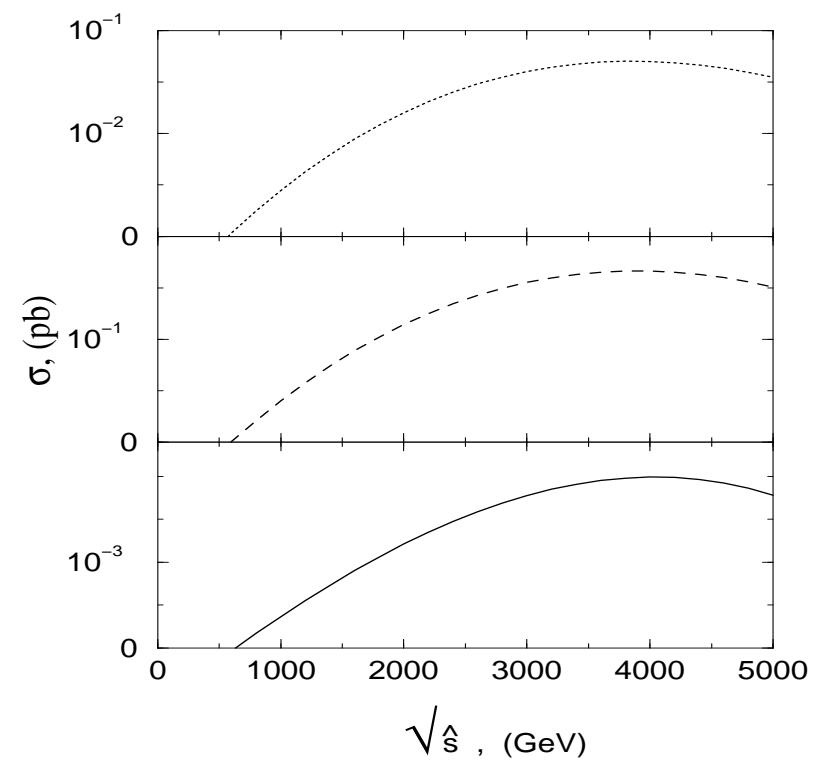

FIG. 4: The cross sections of the subprocess $u \bar{u} \rightarrow \widetilde{\chi}_{2}^{0} \widetilde{\chi}_{2}^{0}$ as a function of $\sqrt{\hat{s}}$. The curves correspond to: solid-Higgsino-like, dashed-gaugino-like and dotted-mixture cases, respectively.

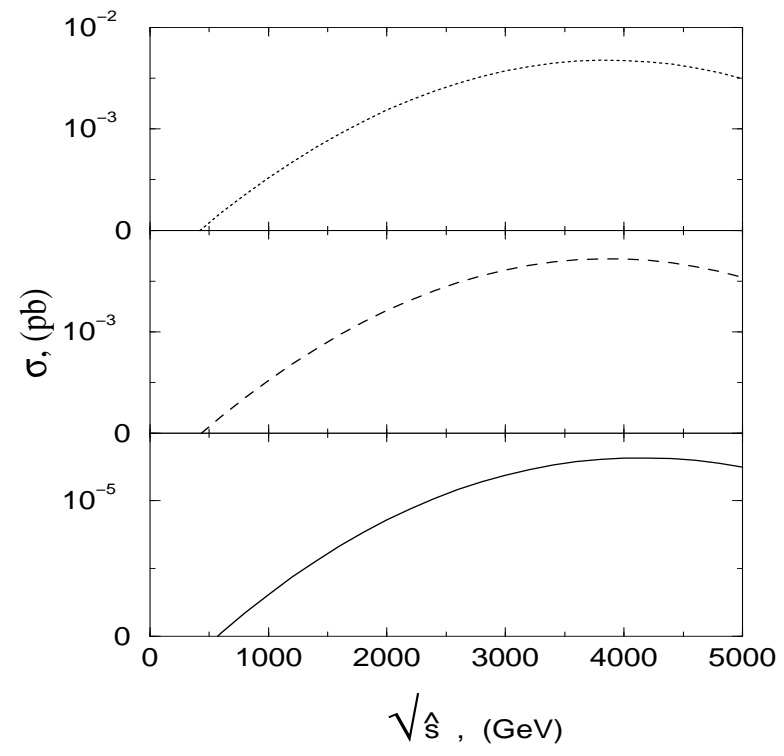

FIG. 5: The cross sections of the subprocess $d \bar{d} \rightarrow \widetilde{\chi}_{1}^{0} \widetilde{\chi}_{1}^{0}$ as a function of $\sqrt{\hat{s}}$. The curves correspond to: solid-Higgsino-like, dashed-gaugino-like and dotted-mixture cases, respectively. 


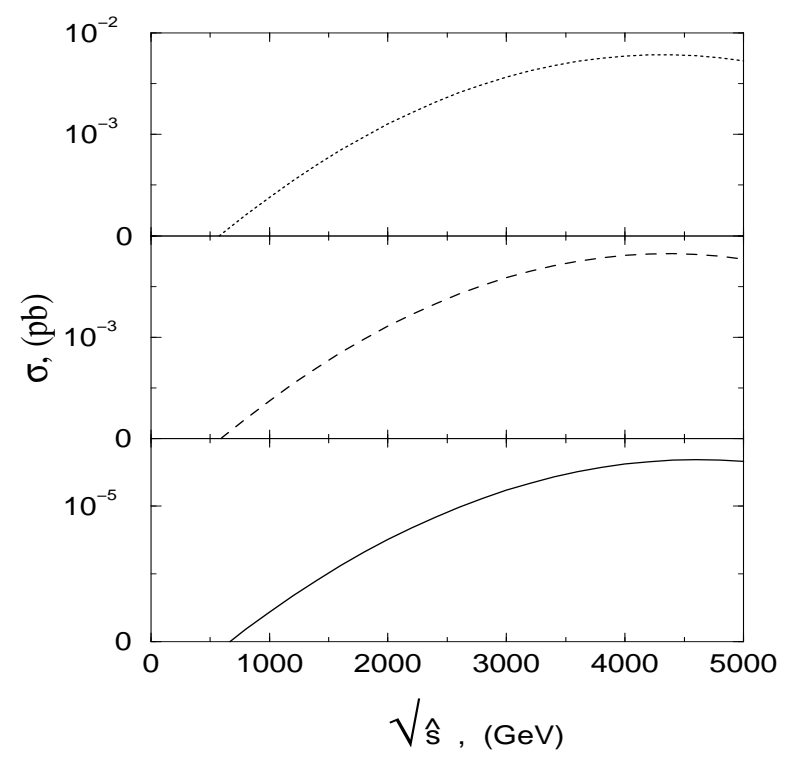

FIG. 6: The cross sections of the subprocess $d \bar{d} \rightarrow \widetilde{\chi}_{1}^{0} \widetilde{\chi}_{2}^{0}$ as a function of $\sqrt{\hat{s}}$. The curves correspond to: solid-Higgsino-like, dashed-gaugino-like and dotted-mixture cases, respectively.

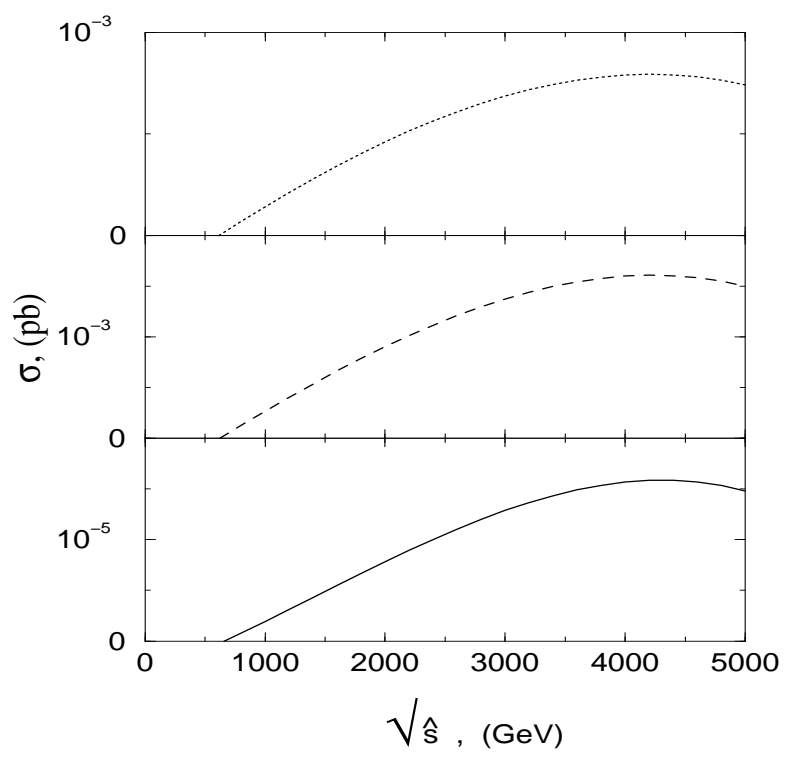

FIG. 7: The cross sections of the subprocess $d \bar{d} \rightarrow \widetilde{\chi}_{2}^{0} \widetilde{\chi}_{2}^{0}$ as a function of $\sqrt{\hat{s}}$. The curves correspond to: solid-Higgsino-like, dashed-gaugino-like and dotted-mixture cases, respectively. 


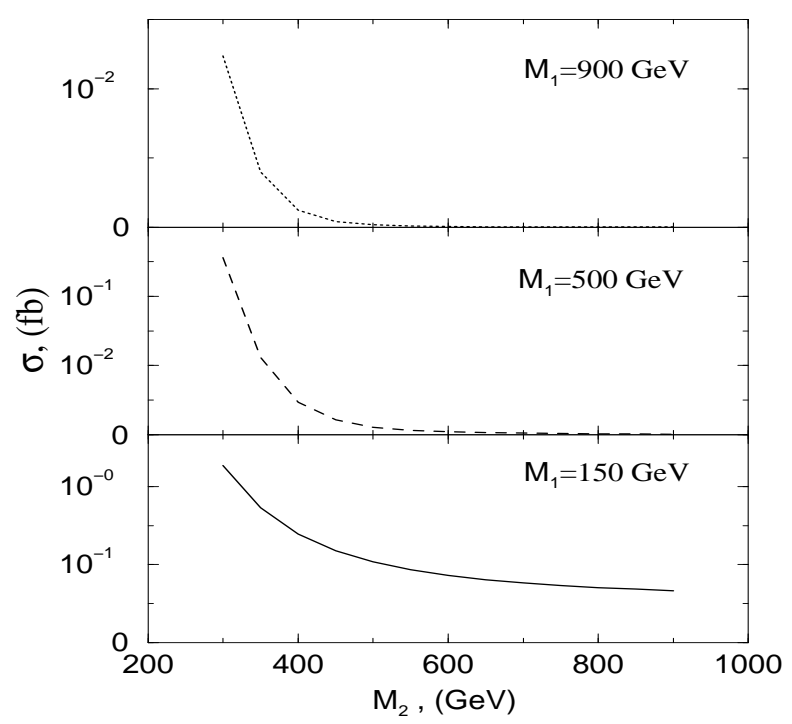

FIG. 8: The cross sections of the subprocess $u \bar{u} \rightarrow \widetilde{\chi}_{1}^{0} \widetilde{\chi}_{1}^{0}$, as a function of $M_{2}$ with $\mu=450 \mathrm{GeV}$ and $\sqrt{\hat{s}}=1.5 \mathrm{TeV}$

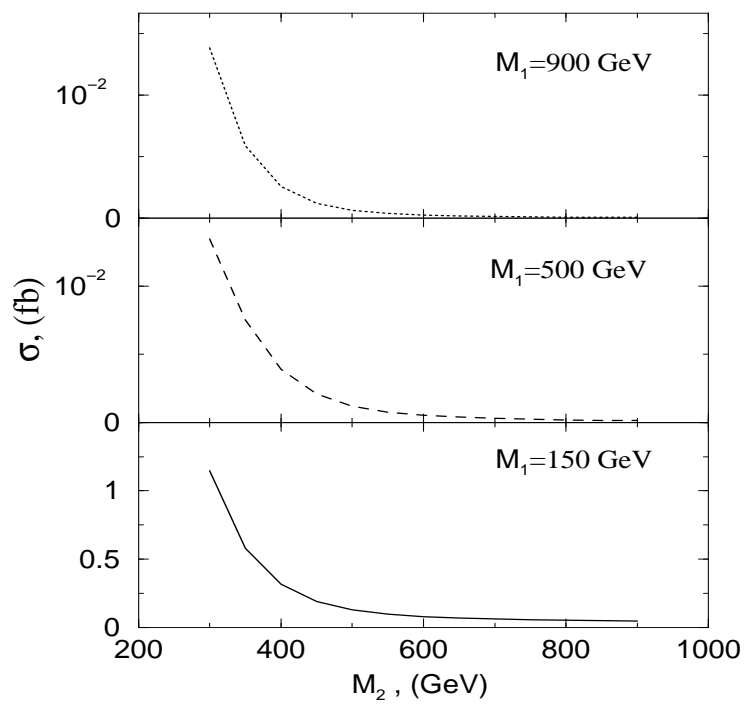

FIG. 9: The cross sections of the subprocess $u \bar{u} \rightarrow \widetilde{\chi}_{1}^{0} \widetilde{\chi}_{2}^{0}$, as a function of $M_{2}$ with $\mu=450 \mathrm{GeV}$ and $\sqrt{\hat{s}}=1.5 \mathrm{TeV}$ 


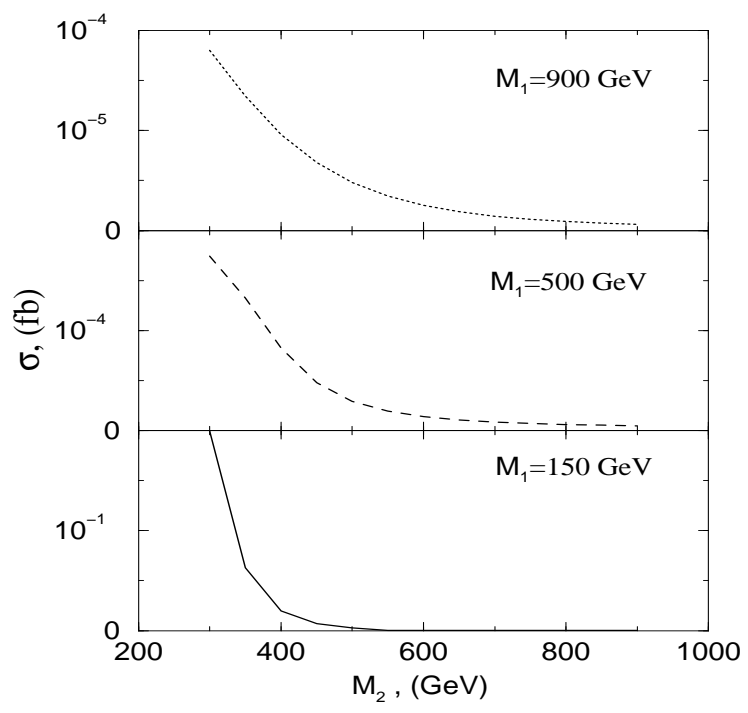

FIG. 10: The cross sections of the subprocess $u \bar{u} \rightarrow \widetilde{\chi}_{2}^{0} \widetilde{\chi}_{2}^{0}$, as a function of $M_{2}$ with $\mu=450 \mathrm{GeV}$ and $\sqrt{\hat{s}}=1.5 \mathrm{TeV}$

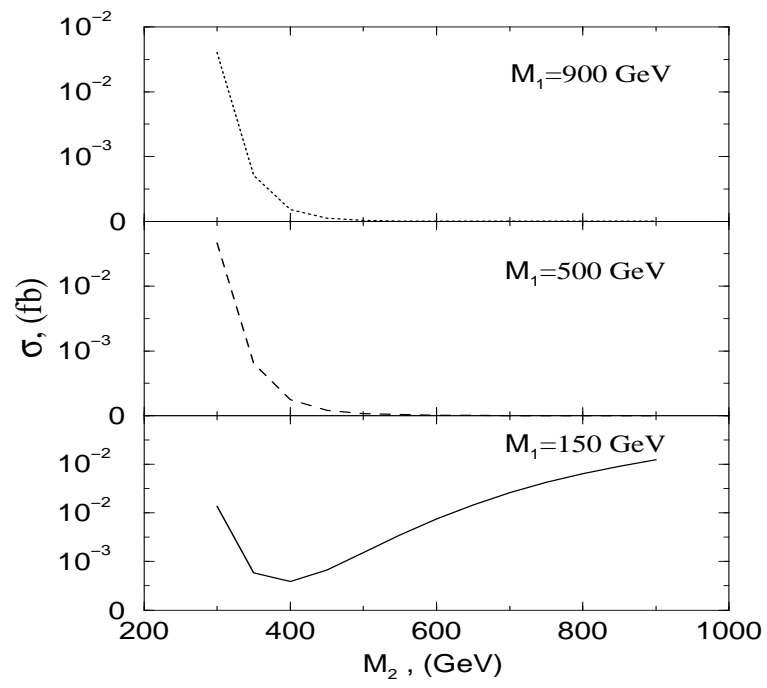

FIG. 11: The cross sections of the subprocess $d \bar{d} \rightarrow \widetilde{\chi}_{1}^{0} \widetilde{\chi}_{1}^{0}$, as a function of $M_{2}$ with $\mu=450 \mathrm{GeV}$ and $\sqrt{\hat{s}}=1.5 \mathrm{TeV}$ 


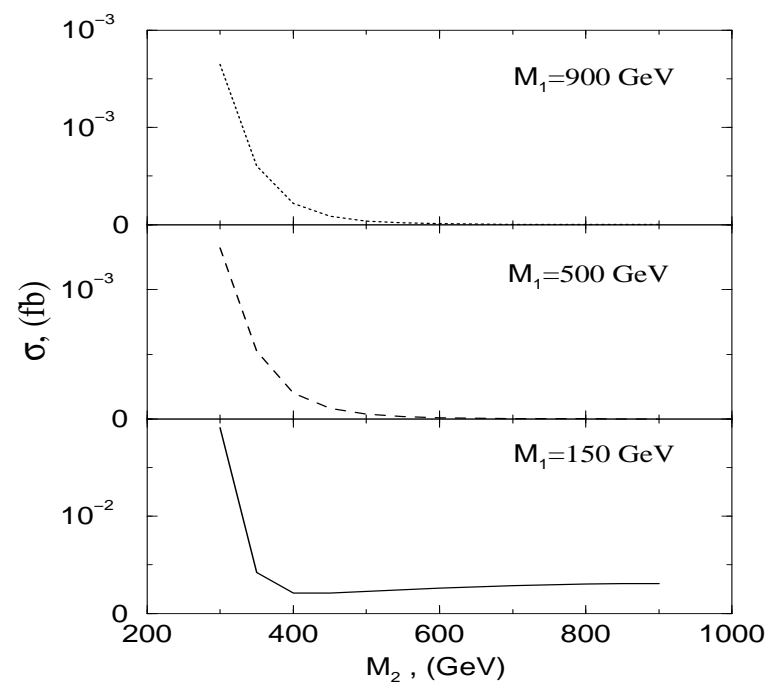

FIG. 12: The cross sections of the subprocess $d \bar{d} \rightarrow \widetilde{\chi}_{1}^{0} \widetilde{\chi}_{2}^{0}$, as a function of $M_{2}$ with $\mu=450 \mathrm{GeV}$ and $\sqrt{\hat{s}}=1.5 \mathrm{TeV}$

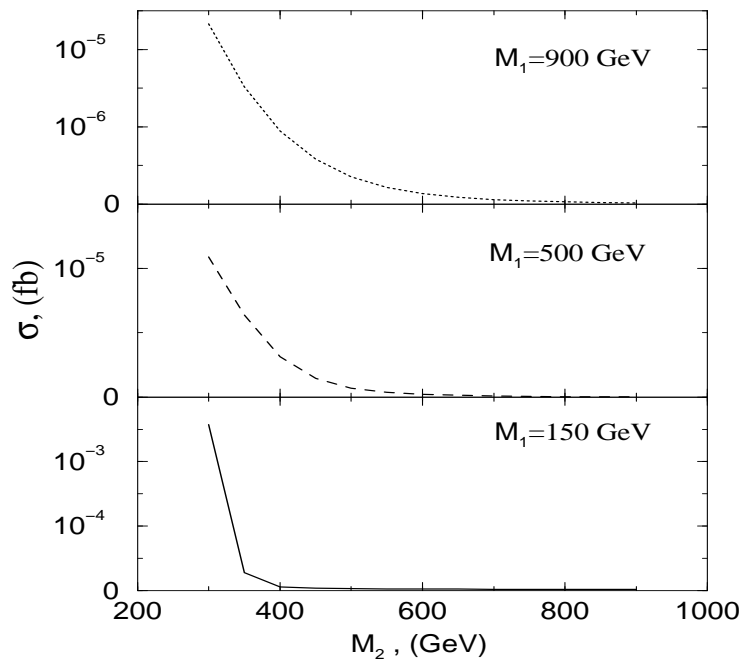

FIG. 13: The cross sections of the subprocess $d \bar{d} \rightarrow \widetilde{\chi}_{2}^{0} \widetilde{\chi}_{2}^{0}$, as a function of $M_{2}$ with $\mu=450 \mathrm{GeV}$ and $\sqrt{\hat{s}}=1.5 \mathrm{TeV}$ 


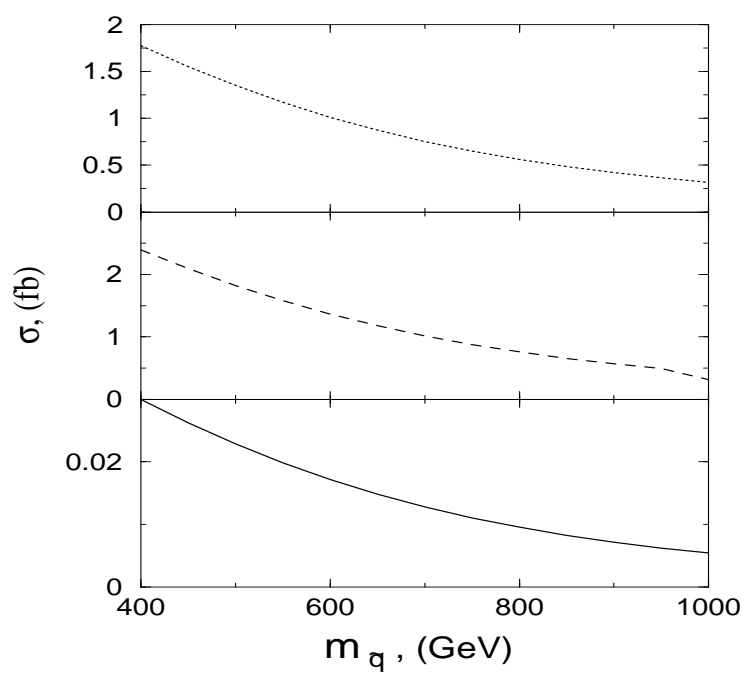

FIG. 14: The cross sections of the subprocess $u \bar{u} \rightarrow \widetilde{\chi}_{1}^{0} \widetilde{\chi}_{1}^{0}$, as a function of the squark mass at beam energy $\sqrt{\hat{s}}=1.5 \mathrm{TeV}$ The curves correspond to: solid-Higgsino-like, dashed-gaugino-like and dotted-mixture cases, respectively.

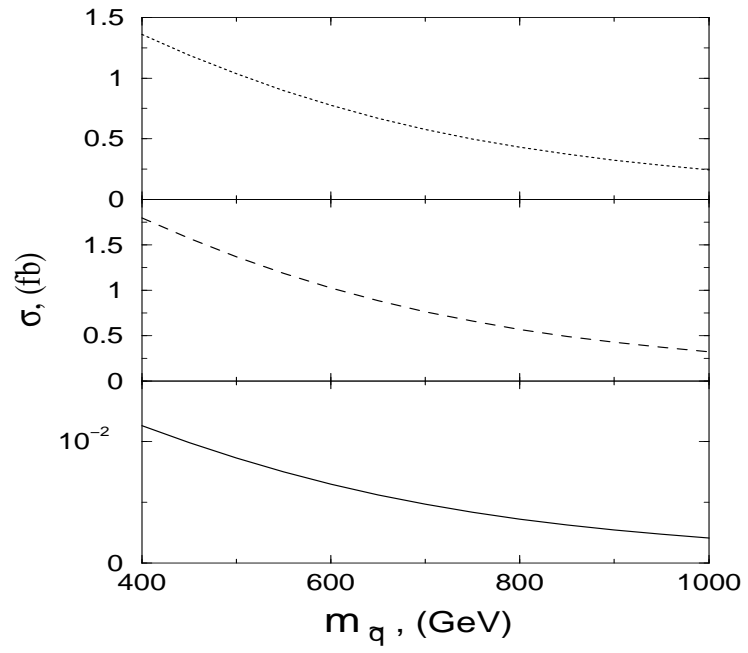

FIG. 15: The cross sections of the subprocess $u \bar{u} \rightarrow \widetilde{\chi}_{1}^{0} \widetilde{\chi}_{2}^{0}$, as a function of the squark mass at beam energy $\sqrt{\hat{s}}=1.5 \mathrm{TeV}$ The curves correspond to: solid-Higgsino-like, dashed-gaugino-like, and dotted-mixture cases, respectively. 


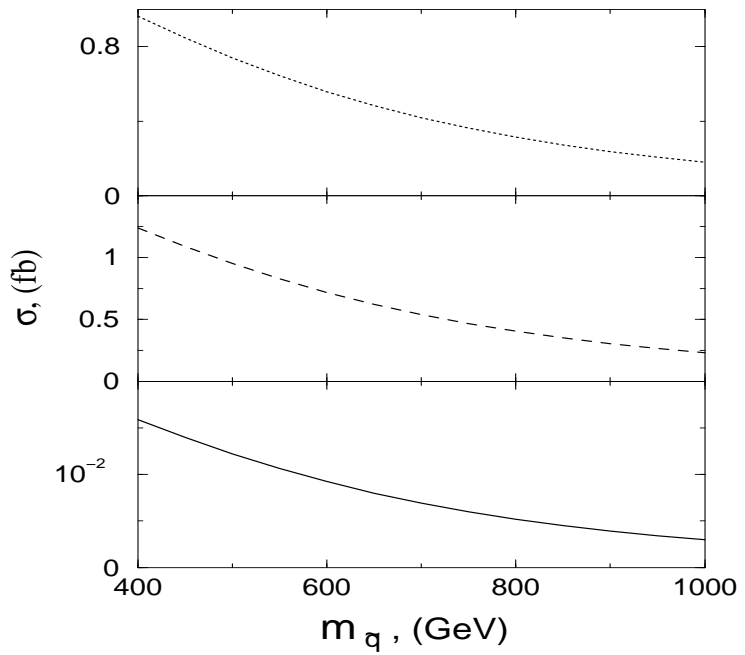

FIG. 16: The cross sections of the subprocess $u \bar{u} \rightarrow \widetilde{\chi}_{2}^{0} \widetilde{\chi}_{2}^{0}$, as a function of the squark mass at beam energy $\sqrt{\hat{s}}=1.5 \mathrm{TeV}$ The curves correspond to: solid-Higgsino-like, dashed-gaugino-like, and dotted-mixture cases, respectively.

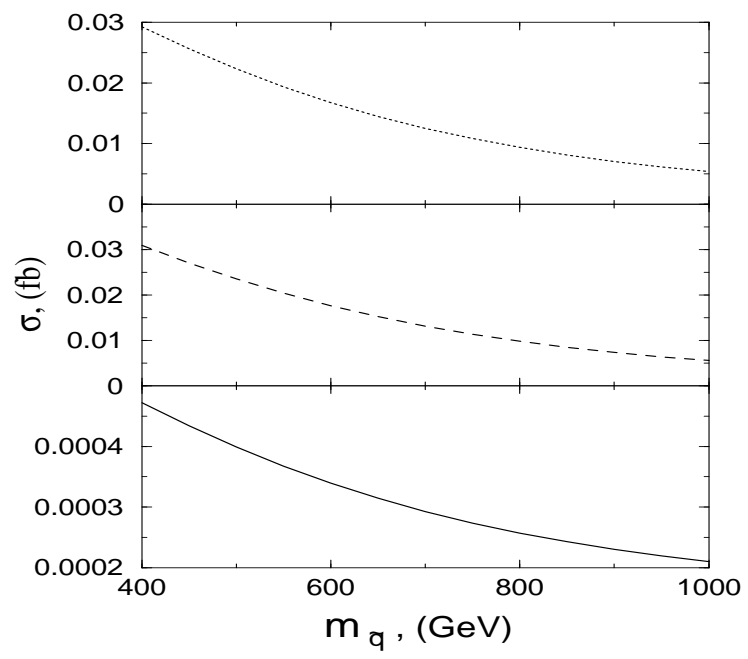

FIG. 17: The cross sections of the subprocess $d \bar{d} \rightarrow \widetilde{\chi}_{1}^{0} \widetilde{\chi}_{1}^{0}$, as a function of the squark mass at beam energy $\sqrt{\hat{s}}=1.5 \mathrm{TeV}$ The curves correspond to: solid-Higgsino-like, dashed-gaugino-like and dotted-mixture cases, respectively. 


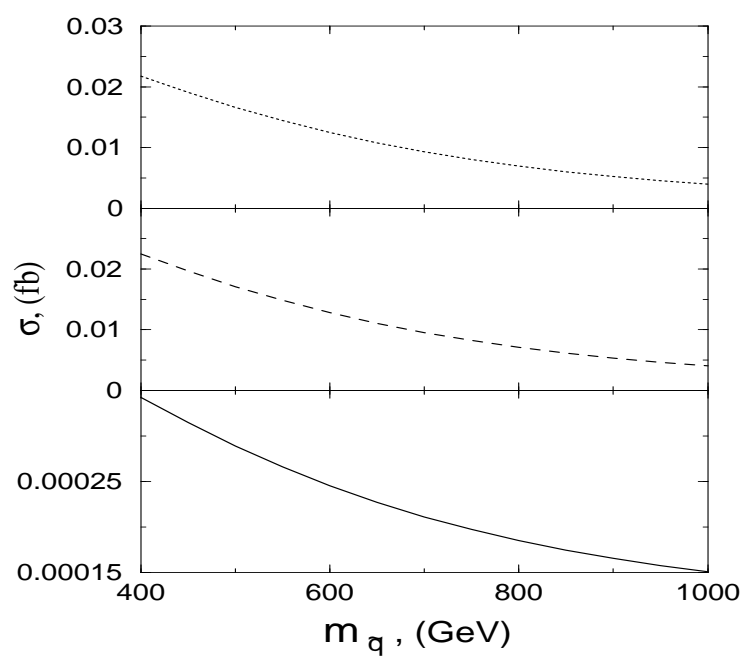

FIG. 18: The cross sections of the subprocess $d \bar{d} \rightarrow \widetilde{\chi}_{1}^{0} \widetilde{\chi}_{2}^{0}$, as a function of the squark mass at beam energy $\sqrt{\hat{s}}=1.5 \mathrm{TeV}$ The curves correspond to: solid-Higgsino-like, dashed-gaugino-like, and dotted-mixture cases respectively.

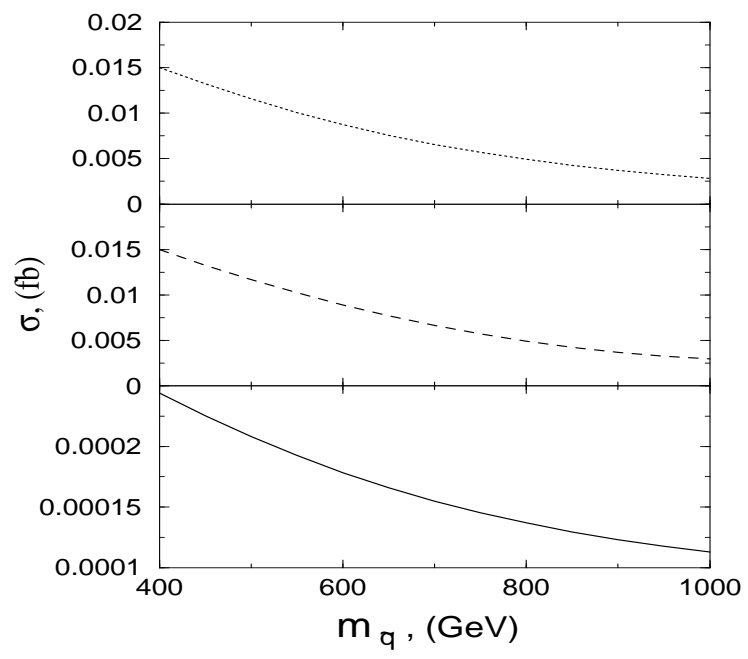

FIG. 19: The cross sections of the subprocess $d \bar{d} \rightarrow \widetilde{\chi}_{2}^{0} \widetilde{\chi}_{2}^{0}$, as a function of the squark mass at beam energy $\sqrt{\hat{s}}=1.5 \mathrm{TeV}$. The curves correspond to: solid-Higgsino-like, dashed-gaugino-like and dotted-mixture cases, respectively. 


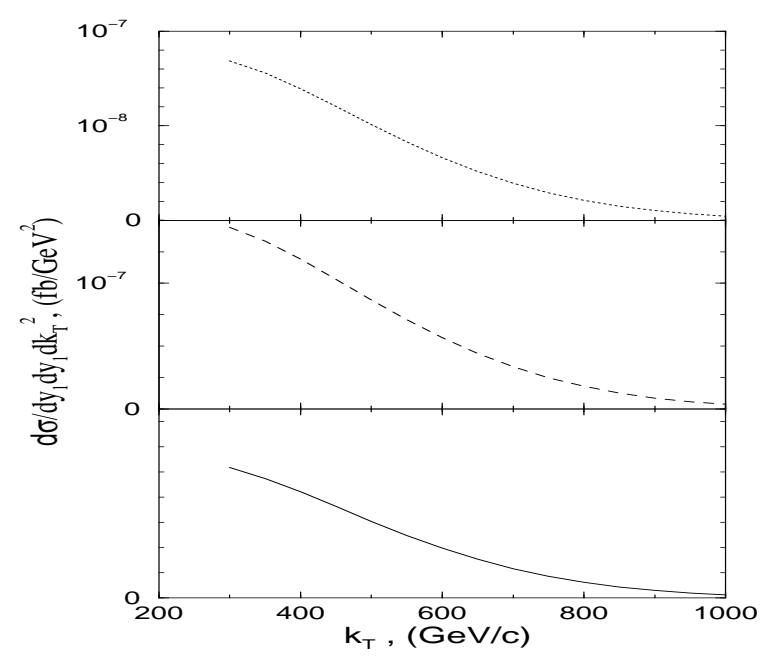

FIG. 20: The differential cross sections of the process $p p \rightarrow \widetilde{\chi}_{1}^{0} \widetilde{\chi}_{1}^{0}$ as a function of the $k_{T}$ transverse momentum of the neutralino pair. The curves correspond to: solid-Higgsino-like, dashed-gauginolike and dotted-mixture cases, respectively.

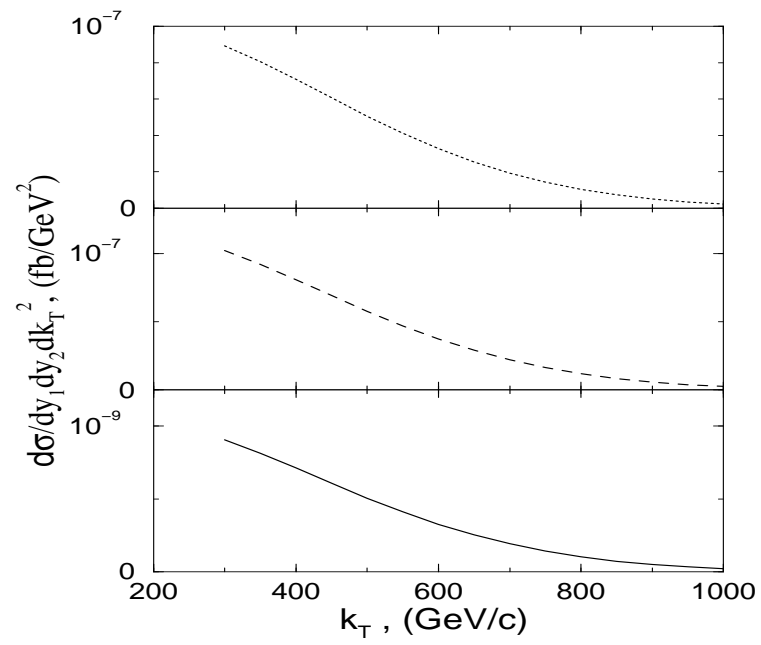

FIG. 21: The differential cross sections of the process $p p \rightarrow \widetilde{\chi}_{1}^{0} \widetilde{\chi}_{2}^{0}$ as a function of the $k_{T}$ transverse momentum of the neutralino pair. The curves correspond to: solid-Higgsino-like, dashed-gauginolike and dotted-mixture cases, respectively. 


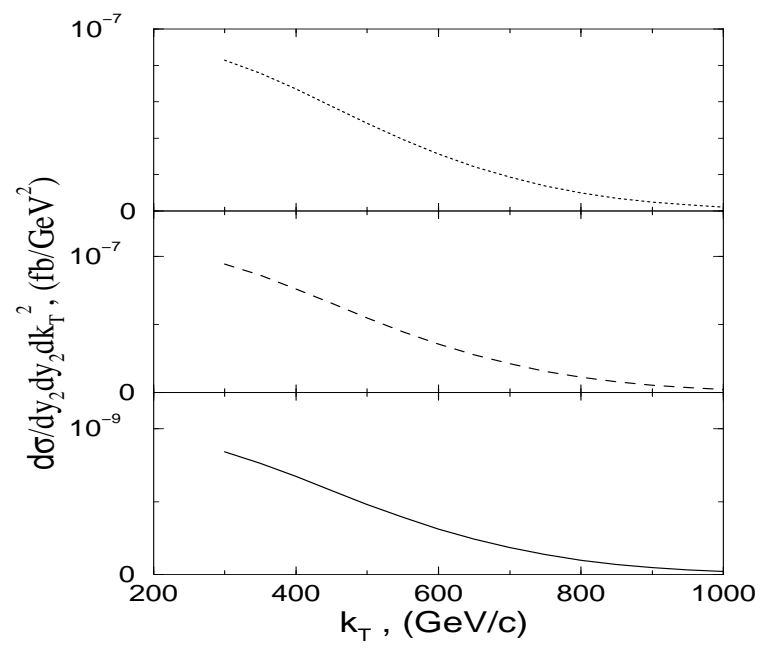

FIG. 22: The differential cross sections of the process $p p \rightarrow \widetilde{\chi}_{2}^{0} \widetilde{\chi}_{2}^{0}$ as a function of the $k_{T}$ transverse momentum of the neutralino pair. The curves correspond to: solid-Higgsino-like, dashed-gauginolike and dotted-mixture cases, respectively. 